\title{
Parametric Design-Based Modal Damped Vibrational Piezoelectric Energy Harvesters with Arbitrary Proof Mass Offset: Numerical and Analytical Validations
}

Mikail F. Lumentut, Ian M. Howard

\author{
Laboratory for Dynamic Systems of Smart Structure and Vibration, \\ Theoretical and Applied Mechanics, Department of Mechanical Engineering \\ Curtin University, Australia
}

Corresponding Author: Email: m.lumentut@exchange.curtin.edu.au, Tel.: +61892667047

\begin{abstract}
The primary contribution of this paper focuses on the development of novel numerical and analytical studies of the modal damped vibration energy harvester using the cantilevered piezoelectric unimorph beam with arbitrary proof mass offset under input base transverse motion. The key equations of electromechanical finite element discretisation for the piezoelectric element with thin electrode layers are revealed and simplified, indicating the most relevant numerical technique in the application for the power harvester research. Full derivations of the electromechanical vibration with damping effects using the extended Lagrangian principle have been developed to give matrix and scalar forms of the coupled system equations. To evaluate the performance of the numerical studies, the analytical closed-form boundary value equations of the physical system have also been developed using the extended Hamiltonian principle. The results from the electromechanical frequency response functions (EFRFs) derived from numerical and analytical studies show excellent agreement with experimental studies. The benefit of numerical techniques is that they can give effective and quick predictions in analysing parametric design optimisation and physical properties for various piezoelectric materials whereas the analytical techniques can provide a very challenging process for developing the derivations and for analysing the complex smart structure. However, the new analytical method presented here shows complete equations of the electromechanical vibration of the piezoelectric structure with dynamical proof mass offset and damping effects providing complementary study for validating the numerical technique. Moreover, the parametric studies using the optimal power harvesting responses enable the identification of the performance for the piezoelectric materials and the particular piezoelectric and proof mass geometries before conducting the micro-fabrication process for emerging micro-sensor power harvesting applications.
\end{abstract}

Keywords: energy harvesting, vibration, electromechanical finite element, closed-form, piezoelectric 


\section{Introduction}

The usage of piezoelectric materials has become important for capturing mechanical energy from the surrounding vibration environment and converting it into electrical energy that enables sensor devices to be completely self-sustaining. Many vibration environments from the machines and infrastructure, including biomechanical human motion, have relatively low frequency vibration excitation that can be used for matching the system response from the piezoelectric structure in order to maximise the power output. For this reason, the development of various mathematical studies has been an important role for modelling electromechanical vibration responses of power harvesters. This includes the comprehensive analytical studies of the optimal power harvesting behaviour with the load resistance using the electromagnetic system [1] and the piezoelectric materials [2]. Moreover, the majority of piezoelectric power harvesters using laminated beams with broad ranges of case studies have been investigated using various analytical techniques such as electromechanical lumped parameter models and electrical equivalent system [3]-[4], analytical approach using weak form techniques [5]-[8], assumed-mode methods [9], transfer matrix method [10] and closed form techniques [11]-[13].

The attached proof mass onto the typical cantilever piezoelectric unimorph and bimorph beams including MEMS devices have been broadly used for high power generation in the lower frequency range, since it can create high elemental strain in the piezoelectric element due to the transverse bending motion for electrical energy generation. Instead of receiving high demand for exploring the recent applications of power harvesting research such as piezo-MEMS devices and the galloping piezoaeroelastic power harvester, the development of the accurate mathematical techniques seems to receive fewer attentions. For example, piezoMEMS power harvesters from previous works generally include experimental studies with oversimplified analytical solution and ignore the dynamical proof mass offset [14]-[16]. Moreover, the piezoaeroelastic power harvesting beam whose dynamic motion is induced from the galloping effect, also ignores the effect of dynamical proof mass offset of the bluff body where the simplified solutions can also be found in the use of lumped parameter models [17]-[18].

Moreover, development of the numerical studies of the electromechanical power harvesting devices has currently received only minor attention. The most notable research articles for the smart structure finite element analysis can be found in studies of piezoelectric 
active control systems. The fundamental concepts of these previous works can be used for the current energy harvesting research studies. The active controlled smart structure system with integrated finite element analysis was formulated using the variational principle [19]- [20]. More details of the active control system using various numerical methods can be found in the published literature reviews [21]. Further active control finite element studies have been extensively investigated using feedback gain control [22], negative velocity feedback control [23] and shunt circuit techniques [24]. In the numerical power harvesting application, the use of piezoelectric material-based ANSYS software with the 3D coupled field solid element has been developed to analyse the electromechanical equivalent circuit parameters where SPICE software was further used for investigating the circuit simulation for power harvesting prediction [25]-[26]. Recently, a new numerical technique of electromechanical finite element vibration modelling which is applicable to the MEMS devices has been developed for predicting power harvesting where the system responses align with the current experimental studies [27].

In this paper, the comprehensive studies of the vibration power harvesters using parametric geometry design and the physical properties of the piezoelectric structures are explored using the proposed two mathematical studies namely, the electromechanical finite element methods and the analytical closed-form boundary value techniques. At this stage, there are no other previous works developing these complete approaches for modelling the parametric identification and optimisation studies. Recent new numerical work from the authors in [27] is extended to outline the key equations and include the damping effects at the beginning of derivation of the electromechanical dynamic equations. This study reveals that the equation-based modal damped vibrations of the electromechanical piezoelectric structure have the technical parameter correlations between mechanical system (elasticity with mechanical stress and internal damping stress, air damping, and dynamic motions), electromechanical system (electrical displacement, electrical stress and electric-polarity field) and electrical system (resistive shunt circuit). These technical correlations can be seen in the development of the electromechanical discretisation (mechanical and electrical discretised element) and formulation of the electromechanical matrix dynamic equations using the Lagrangian principle and the electromechanical scalar dynamic equations for formulating EFRFs. Moreover, the novel analytical studies are also developed using the closed-form boundary value method outlining the functional energy forms using the variational principle in order to derive the integro-differential equations of the piezoelectric structure with 
dynamical proof mass offset. Further technical relations between the numerical and analytical methods can be seen in the use of the same technical parameter correlations while only requiring the change of the local transformation in terms of the kinematic motions of the piezoelectric structure with dynamical proof mass offset. Moreover, the EFRFs from the two methods used in the computational process give very similar result. The benefits of the two methods are also discussed in terms of the level of difficulty, capability, accuracy, and effectiveness. Overall, the proposed two mathematical techniques are compared with each other giving good agreement with the experimental results. The numerical study can be further used for analysing the optimal power harvesting frequency responses and the frequency bandwidth of the parametric geometry design and properties of the piezoelectric materials and proof mass geometries. For this point, the prediction of the power harvesting performance can conveniently be simulated before conducting the fabrication process of the micro-power harvesting sensor device for future applications.

\section{Formulations of Electromechanical Finite Element Vibration System}

The extended linear piezoelectric unimorph beam constitutive equations based on the 3-1 mode of piezoelectric constant operation, 3-3 effect of piezoelectric permittivity and internal damping stress can be formulated as,

$$
\begin{gathered}
\boldsymbol{T}_{1}^{(2)}=\bar{c}_{11}^{(2)}\left(\boldsymbol{S}_{1}^{(2)}+c_{d}^{(2)} \dot{\boldsymbol{S}}_{1}^{(2)}\right)-e_{31} \boldsymbol{E}_{3}, \\
\boldsymbol{D}_{3}=e_{31} \boldsymbol{S}_{1}^{(2)}+\varepsilon_{33}^{S} \boldsymbol{E}_{3}, \\
\varepsilon_{33}^{S}=\varepsilon_{33}^{T}-e_{31} d_{31} \text { or } \varepsilon_{33}^{S}=\varepsilon_{33}^{T}-d_{31}^{2} \bar{c}_{11}^{E} \text { and } e_{31}=d_{31} \bar{c}_{11}^{E} .
\end{gathered}
$$

The linear-elastic constitutive relation for the substructure can also be formulated as,

$$
\boldsymbol{T}_{1}^{(1)}=\bar{c}_{11}^{(1)}\left(\boldsymbol{S}_{1}^{(1)}+c_{d}^{(1)} \dot{\boldsymbol{S}}_{1}^{(1)}\right) .
$$

Note that some parameters as shown in Eqs. (1) and (2) use superscripts 1 and 2 to represent the substructure and piezoelectric layers, respectively. Here, the strain field for each layer of the beam can be formulated as,

$$
S_{1}(x, t)=-z \frac{\partial^{2} w(x, t)}{\partial x^{2}}
$$

where the parameters $\boldsymbol{T}, \boldsymbol{S}, \dot{\boldsymbol{S}}, \boldsymbol{E}$ and $\boldsymbol{D}$ represent stress, strain, strain rate, electric field, and electric displacement, respectively. Moreover, coefficients $c, e$, and $\varepsilon_{33}^{T}$ indicate elastic constant, piezoelectric coefficient, and permittivity at constant strain, respectively. Note that the notations of the piezoelectric structure are written according to the IEEE standards [28]. 
Parameter $c_{d}$ indicates damping coefficient due to internal friction. Variable $z$ is the distance from the neutral axis to each layer.

\subsection{Electromechanical finite element discretisation}

The piezoelectric unimorph considered here consists of piezoelectric and substructure layers including thin electrode layers as shown in Fig. 1. The unimorph structure with arbitrary proof mass offset under base excitation can be connected with the two wires attached on the electrode layers for generating one single voltage output through variable load resistance. In this case, for numerical modelling, the global finite element equations of the system in Fig. 2a-b are based on the mechanical discretised element and the electrical discretised element where this is called the electromechanical discretisation [27].

A few previous research works focusing on the use of proof mass offset on the piezoelectric beam structures have been investigated using different case studies. In [7], the dynamical proof mass offset was analysed using D'Alembert's principle, where other research works with comprehensive analytical piezoelectric beam structure do not provide the concept of obtaining the analytical solution of the proof mass offset [6], [29]. In this paper, dynamics of the arbitrary proof mass offset can be analysed using the rigid-body kinematic equations for formulating the kinetic energy and the non-conservative external work of the system. The benefit of positioning the proof mass with offset distance away from its centroid at the end of the beam is that it can avoid direct contact between the proof mass and the relatively brittle piezoelectric element and the detail of derivations can be found in [27].

Moreover, the solution form of the discretised elemental beam with four-degrees-offreedom as shown in Fig. $2 \mathrm{~b}$ can be formulated using the first-order Hermite interpolation of the cubic relative displacement function to give,

$$
\boldsymbol{w}(x, t)=\boldsymbol{\Phi}^{e}(x) \boldsymbol{u}^{e}(t) .
$$

Parameters of the shape function $\boldsymbol{\Phi}$ and the elemental displacement vector $\boldsymbol{u}$ for each node can be formulated as,

$$
\begin{aligned}
\boldsymbol{\Phi}^{e}(x)= & {\left[\begin{array}{llll}
\Phi_{1}(x) & \Phi_{2}(x) & \Phi_{3}(x) & \Phi_{4}(x)
\end{array}\right], } \\
& \boldsymbol{u}^{e}(t)=\left[\begin{array}{llll}
u_{1} & u_{2} & u_{3} & u_{4}
\end{array}\right]^{\mathrm{T}},
\end{aligned}
$$

where, 


$$
\begin{gathered}
\Phi_{1}(x)=1-3\left(\frac{x-x_{e}}{L_{e}}\right)^{2}+2\left(\frac{x-x_{e}}{L_{e}}\right)^{3}, \Phi_{2}(x)=\left(x-x_{e}\right)\left(1-\frac{x-x_{e}}{L_{e}}\right)^{2}, \\
\Phi_{3}(x)=3\left(\frac{x-x_{e}}{L_{e}}\right)^{2}-2\left(\frac{x-x_{e}}{L_{e}}\right)^{3}, \Phi_{4}(x)=\left(x-x_{e}\right)\left[\left(\frac{x-x_{e}}{L_{e}}\right)^{2}-\frac{x-x_{e}}{L_{e}}\right], \\
L_{e}=x_{e+1}-x_{e}, u_{1}(t)=w_{1}(t), \quad u_{2}(t)=\theta_{1}(t), \\
u_{3}(t)=w_{2}(t), u_{4}(t)=\theta_{2}(t) .
\end{gathered}
$$

The strain-displacement relationship in terms of the vector displacement can be expressed as,

$$
\boldsymbol{S}_{1}(x, t)=-\mathrm{z} \boldsymbol{\Psi}^{e}(x) \boldsymbol{u}^{e}(t),
$$

where the differential form of the shape function $\Psi$ of the strain displacement relationship can be formulated as,

$$
\boldsymbol{\Psi}^{e}(x)=\frac{\mathrm{d}^{2} \boldsymbol{\Phi}^{e}(x)}{\mathrm{d} x^{2}}=\left[\begin{array}{llll}
\Psi_{1}(x) & \Psi_{2}(x) & \Psi_{3}(x) & \Psi_{4}(x)
\end{array}\right]
$$

where $\Psi_{1}(x)=\frac{\mathrm{d}^{2} \Phi_{1}(x)}{\mathrm{d} x^{2}}, \Psi_{2}(x)=\frac{\mathrm{d}^{2} \Phi_{2}(x)}{\mathrm{d} x^{2}}, \Psi_{3}(x)=\frac{\mathrm{d}^{2} \Phi_{3}(x)}{\mathrm{d} x^{2}}, \Psi_{4}(x)=\frac{\mathrm{d}^{2} \Phi_{4}(x)}{\mathrm{d} x^{2}}$.

The discretised electric field $\boldsymbol{E}$ can be assumed to be linear along the thickness of the piezoelectric material for inducing electrical potential $\boldsymbol{\phi}$ over the piezoelectric element. The electric field can be formulated as,

$$
\boldsymbol{E}_{3}=-\nabla \boldsymbol{\phi}^{e}(z, t)=-\boldsymbol{\Omega}^{e}(z) \boldsymbol{\nu}^{(e)}(t),
$$

where $\boldsymbol{\phi}^{e}(z, t)=\boldsymbol{\vartheta}^{e}(z) \boldsymbol{v}^{e}(t)$ is the electrical potential with linear assumption and $\vartheta^{(e)}(z)=\left(z-z_{n}+h_{p}\right) / h_{p}$ is the shape function over the interval $z_{n}-h_{p} \leq z \leq z_{n}$ and $z_{n}=\frac{c_{11}^{(1)} h_{s}{ }^{2}+c_{11}^{(2)} h_{p}{ }^{2}+2 c_{11}^{(1)} h_{s} h_{p}}{2\left(c_{11}^{(1)} h_{s}+c_{11}^{(2)} h_{p}\right)}$ indicates the distance from the asymmetric neutral axis to the top layer of the unimorph. Symbol $\nabla$ is a gradient operator for the first derivative of the shape function with respect to the thickness direction, giving $\boldsymbol{\Omega}^{e}(z)=\mathrm{d} \vartheta^{e}(z) / \mathrm{d} z=1 / h_{p}$.

The stress fields in the partial differential shape function forms can be expressed by substituting Eqs. (7) - (9) into the first part of Eq. (1) and Eq. (2) to give,

$$
\begin{gathered}
\boldsymbol{T}_{1}^{(1)}=-\mathrm{z} \bar{c}_{11}^{(1)} \boldsymbol{\Psi}^{\mathrm{e}}(x) \boldsymbol{u}^{\mathrm{e}}(t)-\mathrm{z} \bar{c}_{11}^{(1)} c_{d}^{(1)} \boldsymbol{\Psi}^{\mathrm{e}}(x) \dot{\boldsymbol{u}}^{\mathrm{e}}(t), \\
\boldsymbol{T}_{1}^{(2)}=-z \bar{c}_{11}^{(2)} \boldsymbol{\Psi}^{e}(x) \boldsymbol{u}^{e}(t)-z \bar{c}_{11}^{(2)} c_{d}^{(2)} \boldsymbol{\Psi}^{e}(x) \dot{\boldsymbol{u}}^{e}(t)+e_{31} \boldsymbol{\Omega}(z) \boldsymbol{\nu}^{e}(t) .
\end{gathered}
$$


The electric displacement vector of the piezoelectric component can be formulated by substituting Eqs. (7)-(9) into the second part of Eq. (1) to give,

$$
\boldsymbol{D}_{3}=-z e_{31} \boldsymbol{\Psi}^{e}(x) \boldsymbol{u}^{e}(t)-\varepsilon_{33}^{S} \boldsymbol{\Omega}^{e}(z) \boldsymbol{v}^{e}(t) \quad .
$$

\subsection{Lagrangian electromechanical finite element equations}

The extended Lagrange equations for deriving the electromechanical discretised finite element dynamic equations of the piezoelectric power harvester can be formulated as,

$$
\begin{gathered}
\frac{\mathrm{d}}{\mathrm{d} t} \frac{\partial \Pi}{\partial \dot{\boldsymbol{u}}^{e}}-\frac{\partial \Pi}{\partial \boldsymbol{u}^{e}}-\boldsymbol{F}_{w}-\boldsymbol{F}_{d}=0, \\
\frac{\mathrm{d}}{\mathrm{d} t} \frac{\partial \Pi}{\partial \dot{\boldsymbol{v}}^{e}}-\frac{\partial \Pi}{\partial \boldsymbol{v}^{e}}-\boldsymbol{F}_{q}=0,
\end{gathered}
$$

where $\Pi=K E-P E+W E, \boldsymbol{F}_{w}=\frac{\partial W F}{\partial \boldsymbol{u}^{e}}, \boldsymbol{F}_{q}=\frac{\partial W F}{\partial \boldsymbol{v}^{e}}$, and $\boldsymbol{F}_{d}=\frac{\partial P D}{\partial \dot{\boldsymbol{u}}^{e}}$.

The extended Lagrangian electromechanical equation can be formulated as,

$$
\left.\begin{array}{l}
\frac{\mathrm{d}}{\mathrm{d} t} \frac{\partial K E}{\partial \dot{\boldsymbol{u}}^{e}}+\frac{\partial P E}{\partial \boldsymbol{u}^{e}}-\frac{\partial W E}{\partial \boldsymbol{u}^{e}}-\frac{\partial W F}{\partial \boldsymbol{u}^{e}}-\frac{\partial P D}{\partial \dot{\boldsymbol{u}}^{e}}=0 \\
\frac{\partial P E}{\partial \boldsymbol{v}^{e}}-\frac{\partial W E}{\partial \boldsymbol{v}^{e}}-\frac{\partial W F}{\partial \boldsymbol{v}^{e}}=0
\end{array}\right\} \begin{gathered}
P E, W E \in\left\{\dot{\boldsymbol{u}}^{e}, \dot{\boldsymbol{v}}^{e}\right\} \text { and } K E, P D \in\left\{\dot{\boldsymbol{u}}^{e}\right\} \\
W F \in\left\{\boldsymbol{u}^{e}, \boldsymbol{v}^{e}\right\}
\end{gathered}
$$

It is important to note here that since the unimorph beam with proof mass offset was operated under input base excitation, the mathematical expressions of the functional energies implied from Eq. (13) were reduced due to the relative displacement $w(x, t)$ defined as the difference between absolute displacement $\boldsymbol{w}_{a b s}(x, t)$ and base excitation $\boldsymbol{w}_{\text {base }}(t)$. The kinetic energy can be formulated from the mass densities of the unimorph layers and proof mass offset as,

$$
\begin{aligned}
& K E=\frac{1}{2} \int_{x_{e}}^{x_{e+1}} \int_{A^{(1)}} \rho^{(1)} \dot{\boldsymbol{w}}(x, t)^{T} \dot{\boldsymbol{w}}(x, t) \mathrm{d} A^{(1)} \mathrm{d} x+\frac{1}{2} \int_{x_{e}}^{x_{e+1}} \int_{A^{(2)}} \rho^{(2)} \dot{\boldsymbol{w}}(x, t)^{T} \dot{\boldsymbol{w}}(x, t) \mathrm{d} A^{(2)} \mathrm{d} x \\
& +I_{0}^{t i p} x_{c} \dot{\boldsymbol{w}}\left(x_{e+1}, t\right)^{T} \dot{\boldsymbol{\theta}}\left(x_{e+1}, t\right)+\frac{1}{2} I_{0}^{t i p} \dot{\boldsymbol{w}}\left(x_{e+1}, t\right)^{T} \dot{\boldsymbol{w}}\left(x_{e+1}, t\right)+\frac{1}{2} I_{2}^{t i p} \dot{\boldsymbol{\theta}}\left(x_{e+1}, t\right)^{T} \dot{\boldsymbol{\theta}}\left(x_{e+1}, t\right) .
\end{aligned}
$$

Note that full derivation of Eq. (14) can be found in [27] where equation (14) excludes rotary inertia effect of the unimorph. Parameters $I_{0}^{\text {tip }}$ and $I_{2}^{\text {tip }}$ can be seen in Appendix A. The potential energy due to stress-strain-electric-damping stress relation for the unimorph layers can be formulated as,

$$
P E=\frac{1}{2} \int_{x_{e}}^{x_{e+1}} \int_{A^{(1)}} \boldsymbol{S}_{1}^{(1)^{T}} \boldsymbol{T}_{1}^{(1)} \mathrm{d} A^{(1)} \mathrm{d} x+\frac{1}{2} \int_{x_{e}}^{x_{e+1}} \int_{A^{(2)}} \boldsymbol{S}_{1}^{(2)^{T}} \boldsymbol{T}_{1}^{(2)} \mathrm{d} A^{(2)} \mathrm{d} x .
$$


The electrical energy term for the piezoelectric element can be formulated as,

$$
W E=\frac{1}{2} \int_{x_{e}}^{x_{e+1}} \int_{A^{(2)}} \boldsymbol{E}_{3}{ }^{T} \boldsymbol{D}_{3} \mathrm{~d} A^{(2)} \mathrm{d} x .
$$

The non-conservative work on the system due to the input base excitation and electrical charge output can be stated as,

$$
\begin{aligned}
W F= & -\int_{x_{e}}^{x_{e+1}} \int_{A^{(1)}} \rho^{(1)} \boldsymbol{w}(x, t)^{T} \mathrm{~d} A^{(1)} \mathrm{d} x \ddot{\boldsymbol{w}}_{\text {base }}(t)-\int_{x_{e}}^{x_{e+1}} \int_{A^{(2)}} \rho^{(2)} \boldsymbol{w}(x, t)^{T} \mathrm{~d} A^{(2)} \mathrm{d} x \ddot{\boldsymbol{w}}_{\text {base }}(t) \\
& -I_{0}^{t i p} x_{c} \boldsymbol{\theta}\left(x_{e+1}, t\right)^{T} \ddot{\boldsymbol{w}}_{\text {base }}(t)-\boldsymbol{I}_{0}^{t i p} \boldsymbol{w}\left(x_{e+1}, t\right)^{T} \ddot{\boldsymbol{w}}_{\text {base }}(t)+\boldsymbol{q}^{(e)}(t) \boldsymbol{v}^{(e)}(t) .
\end{aligned}
$$

The power dissipated by air friction on the unimorph and the proof mass offset using Rayleigh's dissipation function can be stated as,

$$
\begin{aligned}
P D & =-\frac{1}{2} \int_{x_{e}}^{x_{e+1}} \int_{A^{(1)}} c_{v} \rho^{(1)} \dot{\boldsymbol{w}}(x, t)^{T} \dot{\boldsymbol{w}}(x, t) \mathrm{d} A^{(1)} \mathrm{d} x-\frac{1}{2} \int_{x_{e}}^{x_{e+1}} \int_{A^{(2)}} c_{v} \rho^{(2)} \dot{\boldsymbol{w}}(x, t) \dot{\boldsymbol{w}}(x, t) \mathrm{d} A^{(2)} \mathrm{d} x \\
& -c_{v} I_{0}^{t i p} x_{c} \dot{\boldsymbol{\theta}}\left(x_{e+1}, t\right) \dot{\boldsymbol{w}}\left(x_{e+1}, t\right)-\frac{1}{2} c_{v} I_{0}^{t i p} \dot{\boldsymbol{w}}\left(x_{e+1}, t\right) \dot{\boldsymbol{w}}\left(x_{e+1}, t\right)-\frac{1}{2} c_{v} I_{2}^{t i p} \dot{\boldsymbol{\theta}}\left(x_{e+1}, t\right) \dot{\boldsymbol{\theta}}\left(x_{e+1}, t\right) .
\end{aligned}
$$

Note that since the structure is under dynamic motion, the damping coefficient due to air friction $c_{v}$ is also considered. So far, two damping coefficients have been introduced into the system as presented in Eqs. (1), (10) and (18). The power dissipation due to air damping occurs due to the kinetic energy of the structure at particular times creating air friction. The expressions given from Eqs. (3), (7), (8), (10) and (11) can be substituted into Eqs. (14)-(18) to give two electromechanical dynamic equations using Eq. (12). After simplifying, the first damped electromechanical dynamic equation due to the transverse bending form can be expressed as,

$$
\begin{gathered}
\int_{x_{e}}^{x_{e+1}} \int_{A^{(1)}} \rho^{(1)} \boldsymbol{\Phi}^{e}(x)^{T} \boldsymbol{\Phi}^{e}(x) \ddot{u}^{e}(t) \mathrm{d} A^{(1)} \mathrm{d} x+\int_{x_{e}}^{x_{e+1}} \int^{(2)} \rho^{(2)} \boldsymbol{\Phi}^{e}(x)^{T} \boldsymbol{\Phi}^{(e)}(x) \ddot{\boldsymbol{u}}^{(e)}(t) \mathrm{d} A^{(2)} \mathrm{d} x \\
+2 I_{0}^{t i p} x_{c} \boldsymbol{\Phi}^{e}\left(x_{e+1}\right)^{T} \frac{\mathrm{d} \boldsymbol{\Phi}^{e}\left(x_{e+1}\right)}{\mathrm{d} x} \ddot{\boldsymbol{u}}(t)+I_{0}^{t i p} \boldsymbol{\Phi}^{e}\left(x_{e+1}\right)^{T} \boldsymbol{\Phi}^{e}\left(x_{e+1}\right) \ddot{\boldsymbol{u}}^{e}(t)+I_{2}^{t i p} \frac{\mathrm{d} \boldsymbol{\Phi}^{e}\left(x_{e+1}\right)^{T}}{\mathrm{~d} x} \frac{\mathrm{d} \boldsymbol{\Phi}^{e}\left(x_{e+1}\right)}{\mathrm{d} x} \ddot{\boldsymbol{u}}^{e}(t) \\
+z^{2} \bar{c}_{11}^{(1)} c_{d}^{(1)} \boldsymbol{\Psi}^{e}(x) \boldsymbol{\Psi}^{e}(x)^{T} \dot{\boldsymbol{u}}^{e}(t)+z^{2} \bar{c}_{11}^{(2)} c_{d}^{(2)} \boldsymbol{\Psi}^{e}(x) \boldsymbol{\Psi}^{e}(x)^{T} \dot{\boldsymbol{u}}^{e}(t)+\int_{x_{e}}^{x_{e+1}} \int_{A^{(1)}} \rho^{(1)} \boldsymbol{\Phi}^{e}(x)^{T} c_{v} \boldsymbol{\Phi}^{e}(x) \dot{\boldsymbol{u}}^{e}(t) \mathrm{d} A^{(1)} \mathrm{d} x \\
+\int_{x_{e}}^{x_{e+1}} \int_{A^{(2)}} \rho^{(2)} \boldsymbol{\Phi}^{e}(x)^{T} c_{v} \boldsymbol{\Phi}^{e}(x) \dot{\boldsymbol{u}}^{e}(t) \mathrm{d} A^{(1)} \mathrm{d} x+2 I_{0}^{t i p} x_{c} c_{v} \boldsymbol{\Phi}^{e}\left(x_{e+1}\right)^{T} \frac{d \boldsymbol{\Phi}^{e}\left(x_{e+1}\right)}{d \mathrm{x}} \ddot{\boldsymbol{u}}^{e}(t) \\
+I_{0}^{t i p} c_{v} \boldsymbol{\Phi}^{e}\left(x_{e+1}\right)^{\mathbf{T}} \boldsymbol{\Phi}^{e}\left(x_{e+1}\right) \ddot{\boldsymbol{u}}^{e}(t)+I_{2}^{t i p} c_{v} \frac{d \boldsymbol{\Phi}^{e}\left(x_{e+1}\right)^{T}}{d x} \frac{d \boldsymbol{\Phi}^{e}\left(x_{e+1}\right)}{d x} \ddot{\boldsymbol{u}}^{e}(t)
\end{gathered}
$$




$$
\begin{aligned}
& +\int_{x_{e}}^{x_{e+1}} \int_{A^{(1)}} z^{2} \bar{c}_{11}^{(1)} \boldsymbol{\Psi}^{e}(x) \boldsymbol{\Psi}^{e}(x)^{T} \boldsymbol{u}^{e}(t) \mathrm{d} A^{(1)} \mathrm{d} x+\int_{x_{e}}^{x_{e+1}} \int_{A^{(2)}} z^{2} \bar{c}_{11}^{(2)} \boldsymbol{\Psi}^{e}(x) \boldsymbol{\Psi}^{e}(x)^{T} u^{e}(t) \mathrm{d} A^{(2)} \mathrm{d} x \\
& -\frac{1}{2} \int_{x_{e}}^{x_{e+1}} \int_{A^{(2)}} z e_{3 l} \boldsymbol{\Psi}^{e}(x)^{T} \boldsymbol{\Omega}^{e}(z) \nu^{e}(t) \mathrm{d} A^{(2)} \mathrm{d} x-\frac{1}{2} \int_{x_{e}}^{x_{e+1}} \int_{A^{(2)}} z e_{31} \boldsymbol{\Psi}^{e}(x)^{T} \boldsymbol{\Omega}^{e}(z) v^{e}(t) \mathrm{d} A^{(2)} \mathrm{d} x \\
& =-\int_{x_{e}}^{x_{e+1}} \int_{A^{(1)}} \rho^{(1)} \boldsymbol{\Phi}^{e}(x)^{T} \mathrm{~d} A^{(1)} \mathrm{d} x \ddot{w}_{\text {base }}(t)-\int_{x_{e}}^{x_{e+1}} \int_{A^{(2)}} \rho^{(2)} \boldsymbol{\Phi}^{e}(x)^{T} \mathrm{~d} A^{(2)} \mathrm{d} x \ddot{w}_{\text {base }}(t) \\
& -I_{0}^{t i p} x_{c} \frac{\mathrm{d} \boldsymbol{\Phi}^{e}\left(L_{e}\right)^{T}}{\mathrm{~d} x} \ddot{w}_{\text {base }}(t)-I_{0}^{t i p} \boldsymbol{\Phi}^{e}\left(L_{e}\right)^{T} \ddot{w}_{\text {base }}(t) .
\end{aligned}
$$

The second electromechanical dynamic equation due to the electrical form can be expressed as,

$$
\begin{aligned}
& -\frac{1}{2} \int_{x_{e}}^{x_{e+1}} \int_{A^{(2)}} z e_{31} \boldsymbol{\Omega}^{e}(z)^{T} \boldsymbol{\Psi}^{e}(x) \boldsymbol{u}^{e}(t) \mathrm{d} A^{(2)} \mathrm{d} x-\frac{1}{2} \int_{x_{e}}^{x_{e+1}} \int_{A^{(2)}} z e_{31} \boldsymbol{\Omega}^{e}(z)^{T} \boldsymbol{\Psi}^{e}(x) \boldsymbol{u}^{e}(t) \mathrm{d} A^{(2)} \mathrm{d} x \\
& -\int_{x_{e}}^{x_{e+1}} \int_{A^{(2)}} \varepsilon_{33} \boldsymbol{\Omega}(z)^{T} \boldsymbol{\Omega}(z) \boldsymbol{v}(t) \mathrm{d} A^{(2)} \mathrm{d} x-\boldsymbol{q}^{e}(t)=0 .
\end{aligned}
$$

Equation (20) can be modified by differentiating with respect to time to give,

$$
\begin{aligned}
& -\frac{1}{2} \int_{x_{e}}^{x_{e+1}} \int_{A^{(2)}} z \boldsymbol{\Omega}(z)^{T} e_{31} \boldsymbol{\Psi}(x) \dot{\boldsymbol{u}}(t) \mathrm{d} A^{(2)} \mathrm{d} x-\frac{1}{2} \int_{x_{e}}^{x_{e+1}} \int_{A^{(2)}} z \boldsymbol{\Omega}(z)^{T} e_{31} \boldsymbol{\Psi}(x) \dot{\boldsymbol{u}}(t) \mathrm{d} A^{(2)} \mathrm{d} x \\
& -\int_{x_{e}}^{x_{e+1}} \int_{A^{(2)}} \varepsilon_{33}^{S} \boldsymbol{\Omega}(z)^{T} \boldsymbol{\Omega}(z) \dot{v}(t) \mathrm{d} A^{(2)} \mathrm{d} x-\boldsymbol{i}_{p}^{e}(t)=0 .
\end{aligned}
$$

The expressions given from Eqs. (19) and (21) can be further simplified to give the local element matrices of damped electromechanical dynamic equations as,

$$
\left[\begin{array}{cc}
\boldsymbol{M}^{e} & 0 \\
0 & 0
\end{array}\right]\left\{\begin{array}{c}
\ddot{\boldsymbol{u}}^{e}(t) \\
\ddot{\boldsymbol{v}}^{e}(t)
\end{array}\right\}+\left[\begin{array}{cc}
\boldsymbol{C}^{e} & 0 \\
\boldsymbol{P}_{\theta}^{e^{T}} & \boldsymbol{P}_{D}^{e}
\end{array}\right]\left\{\begin{array}{c}
\dot{\boldsymbol{u}}^{e}(t) \\
\dot{\boldsymbol{v}}^{e}(t)
\end{array}\right\}+\left[\begin{array}{cc}
\boldsymbol{K}^{e} & \boldsymbol{P}_{\theta}^{e} \\
0 & 0
\end{array}\right]\left\{\begin{array}{c}
\boldsymbol{u}^{e}(t) \\
\boldsymbol{v}^{e}(t)
\end{array}\right\}=\left\{\begin{array}{l}
\boldsymbol{F}^{e} \\
\boldsymbol{i}_{p}^{e}
\end{array}\right\},
$$

where

$$
\begin{gathered}
\boldsymbol{M}^{e}=\sum_{i=1}^{n}\left(\rho^{(i)} A^{(i)} \int_{x_{e}}^{x_{e+1}}\left[\begin{array}{cccc}
\Phi_{1}(x)^{2} & \Phi_{1}(x) \Phi_{2}(x) & \Phi_{1}(x) \Phi_{3}(x) & \Phi_{1}(x) \Phi_{4}(x) \\
\Phi_{2}(x) \Phi_{1}(x) & \Phi_{2}(x)^{2} & \Phi_{2}(x) \Phi_{3}(x) & \Phi_{2}(x) \Phi_{4}(x) \\
\Phi_{3}(x) \Phi_{1}(x) & \Phi_{3}(x) \Phi_{2}(x) & \Phi_{3}(x)^{2} & \Phi_{3}(x) \Phi_{4}(x) \\
\Phi_{4}(x) \Phi_{1}(x) & \Phi_{4}(x) \Phi_{2}(x) & \Phi_{4}(x) \Phi_{3}(x) & \Phi_{4}(x)^{2}
\end{array}\right] \mathrm{d} x\right. \\
+I_{0}^{t i p}\left[\begin{array}{cccc}
\Phi_{1}\left(x_{e+1}\right)^{2} & \Phi_{1}\left(x_{e+1}\right) \Phi_{2}\left(x_{e+1}\right) & \Phi_{1}\left(x_{e+1}\right) \Phi_{3}\left(x_{e+1}\right) & \Phi_{1}\left(x_{e+1}\right) \Phi_{4}\left(x_{e+1}\right) \\
\Phi_{2}\left(x_{e+1}\right) \Phi_{1}\left(x_{e+1}\right) & \Phi_{2}\left(x_{e+1}\right)^{2} & \Phi_{2}\left(x_{e+1}\right) \Phi_{3}\left(x_{e+1}\right) & \Phi_{2}\left(x_{e+1}\right) \Phi_{4}\left(x_{e+1}\right) \\
\Phi_{3}\left(x_{e+1}\right) \Phi_{1}\left(x_{e+1}\right) & \Phi_{3}\left(x_{e+1}\right) \Phi_{2}\left(x_{e+1}\right) & \Phi_{3}\left(x_{e+1}\right)^{2} & \Phi_{3}\left(x_{e+1}\right) \Phi_{4}\left(x_{e+1}\right) \\
\Phi_{4}\left(x_{e+1}\right) \Phi_{1}\left(x_{e+1}\right) & \Phi_{4}\left(x_{e+1}\right) \Phi_{2}\left(x_{e+1}\right) & \Phi_{4}\left(x_{e+1}\right) \Phi_{3}\left(x_{e+1}\right) & \Phi_{4}\left(x_{e+1}\right)^{2}
\end{array}\right]
\end{gathered}
$$




$$
+2 I_{0}^{t i p} x_{c}\left[\begin{array}{llll}
\Phi_{1}\left(x_{e+1}\right) \frac{\mathrm{d} \Phi_{1}\left(x_{e+1}\right)}{\mathrm{d} x} & \Phi_{1}\left(x_{e+1}\right) \frac{\mathrm{d} \Phi_{2}\left(x_{e+1}\right)}{\mathrm{d} x} & \Phi_{1}\left(x_{e+1}\right) \frac{\mathrm{d} \Phi_{3}\left(x_{e+1}\right)}{\mathrm{d} x} & \Phi_{1}\left(x_{e+1}\right) \frac{\mathrm{d} \Phi_{4}\left(x_{e+1}\right)}{\mathrm{d} x} \\
\Phi_{2}\left(x_{e+1}\right) \frac{\mathrm{d} \Phi_{1}\left(x_{e+1}\right)}{\mathrm{d} x} & \Phi_{2}\left(x_{e+1}\right) \frac{\mathrm{d} \Phi_{2}\left(x_{e+1}\right)}{\mathrm{d} x} & \Phi_{2}\left(x_{e+1}\right) \frac{\mathrm{d} \Phi_{3}\left(x_{e+1}\right)}{\mathrm{d} x} & \Phi_{2}\left(x_{e+1}\right) \frac{\mathrm{d} \Phi_{4}\left(x_{e+1}\right)}{\mathrm{d} x} \\
\Phi_{3}\left(x_{e+1}\right) \frac{\mathrm{d} \Phi_{1}\left(x_{e+1}\right)}{\mathrm{d} x} & \Phi_{3}\left(x_{e+1}\right) \frac{\mathrm{d} \Phi_{2}\left(x_{e+1}\right)}{\mathrm{d} x} & \Phi_{3}\left(x_{e+1}\right) \frac{\mathrm{d} \Phi_{3}\left(x_{e+1}\right)}{\mathrm{d} x} & \Phi_{3}\left(x_{e+1}\right) \frac{\mathrm{d} \Phi_{3}\left(x_{e+1}\right)}{\mathrm{d} x} \\
\Phi_{4}\left(x_{e+1}\right) \frac{\mathrm{d} \Phi_{1}\left(x_{e+1}\right)}{\mathrm{d} x} & \Phi_{4}\left(x_{e+1}\right) \frac{\mathrm{d} \Phi_{2}\left(x_{e+1}\right)}{\mathrm{d} x} & \Phi_{4}\left(x_{e+1}\right) \frac{\mathrm{d} \Phi_{3}\left(x_{e+1}\right)}{\mathrm{d} x} & \Phi_{4}\left(x_{e+1}\right) \frac{\mathrm{d} \Phi_{4}\left(x_{e+1}\right)}{\mathrm{d} x}
\end{array}\right]
$$

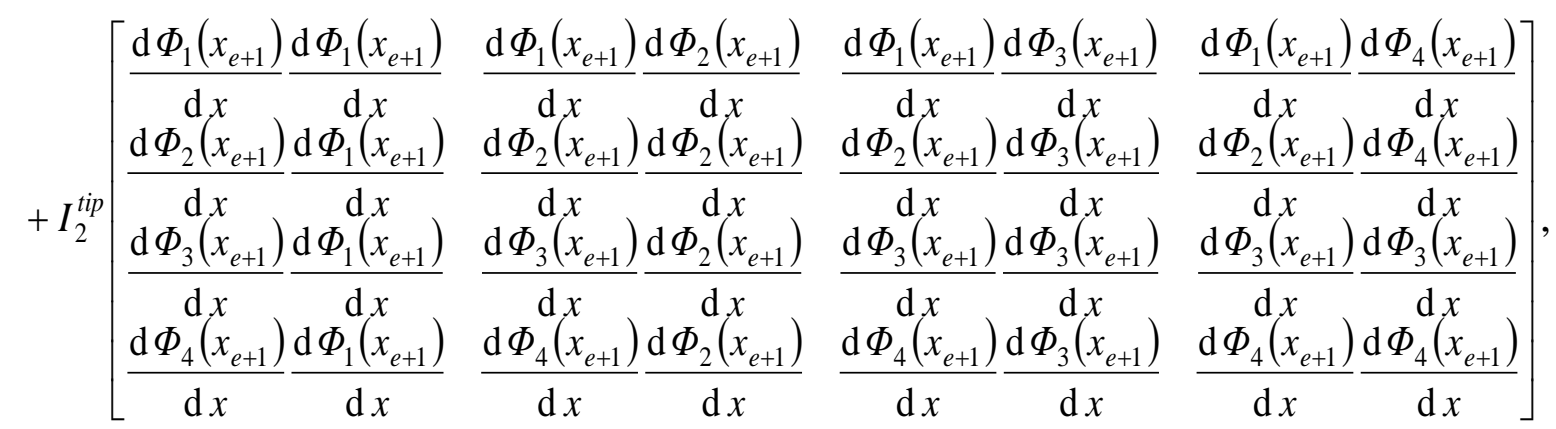

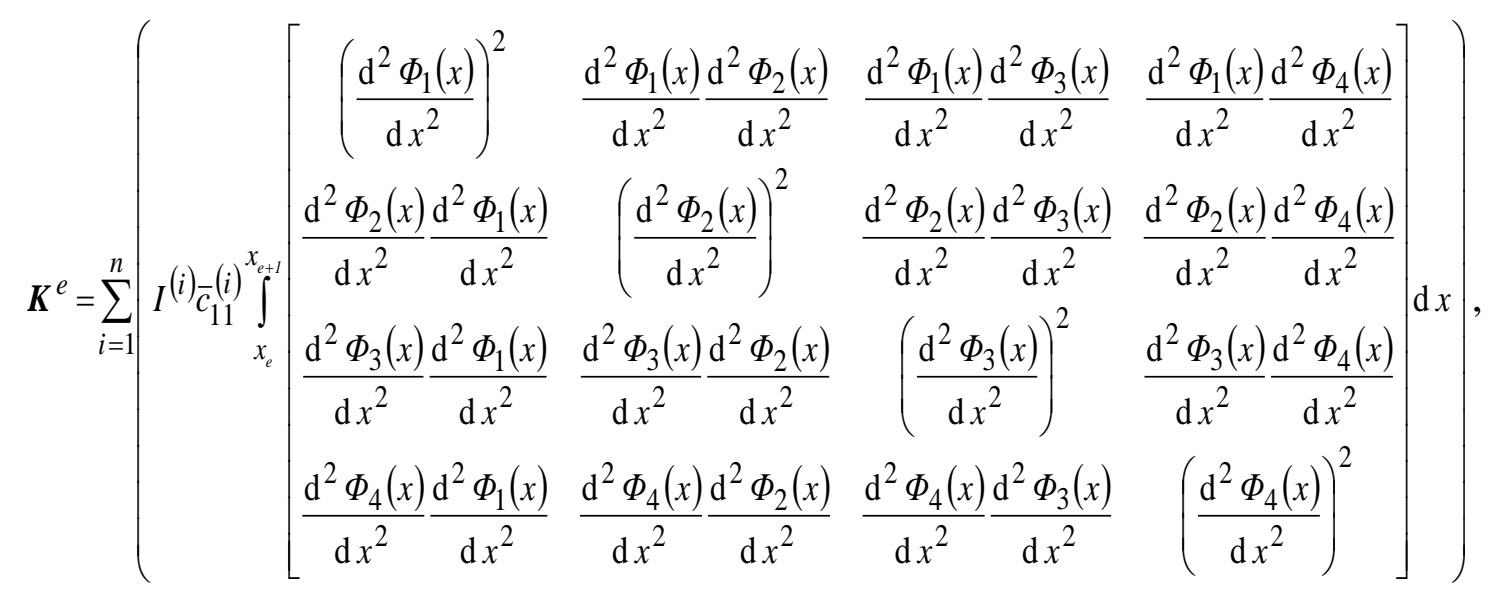$$
\boldsymbol{C}^{e}=\sum_{i=1}^{n}\left(c_{v} \rho^{(i)} A^{(i)} \int_{x_{e}}^{x_{e+1}}\left[\begin{array}{cccc}
\Phi_{1}(x)^{2} & \Phi_{1}(x) \Phi_{2}(x) & \Phi_{1}(x) \Phi_{3}(x) & \Phi_{1}(x) \Phi_{4}(x) \\
\Phi_{2}(x) \Phi_{1}(x) & \Phi_{2}(x)^{2} & \Phi_{2}(x) \Phi_{3}(x) & \Phi_{2}(x) \Phi_{4}(x) \\
\Phi_{3}(x) \Phi_{1}(x) & \Phi_{3}(x) \Phi_{2}(x) & \Phi_{3}(x)^{2} & \Phi_{3}(x) \Phi_{4}(x) \\
\Phi_{4}(x) \Phi_{1}(x) & \Phi_{4}(x) \Phi_{2}(x) & \Phi_{4}(x) \Phi_{3}(x) & \Phi_{4}(x)^{2}
\end{array}\right]\right)
$$$$
+c_{v} I_{0}^{t i p}\left[\begin{array}{cccc}
\Phi_{1}\left(x_{e+1}\right)^{2} & \Phi_{1}\left(x_{e+1}\right) \Phi_{2}\left(x_{e+1}\right) & \Phi_{1}\left(x_{e+1}\right) \Phi_{3}\left(x_{e+1}\right) & \Phi_{1}\left(x_{e+1}\right) \Phi_{4}\left(x_{e+1}\right) \\
\Phi_{2}\left(x_{e+1}\right) \Phi_{1}\left(x_{e+1}\right) & \Phi_{2}\left(x_{e+1}\right)^{2} & \Phi_{2}\left(x_{e+1}\right) \Phi_{3}\left(x_{e+1}\right) & \Phi_{2}\left(x_{e+1}\right) \Phi_{4}\left(x_{e+1}\right) \\
\Phi_{3}\left(x_{e+1}\right) \Phi_{1}\left(x_{e+1}\right) & \Phi_{3}\left(x_{e+1}\right) \Phi_{2}\left(x_{e+1}\right) & \Phi_{3}\left(x_{e+1}\right)^{2} & \Phi_{3}\left(x_{e+1}\right) \Phi_{4}\left(x_{e+1}\right) \\
\Phi_{4}\left(x_{e+1}\right) \Phi_{1}\left(x_{e+1}\right) & \Phi_{4}\left(x_{e+1}\right) \Phi_{2}\left(x_{e+1}\right) & \Phi_{4}\left(x_{e+1}\right) \Phi_{3}\left(x_{e+1}\right) & \Phi_{4}\left(x_{e+1}\right)^{2}
\end{array}\right]
$$ 


$$
+2 c_{v} I_{0}^{i p} x_{c}\left[\begin{array}{llll}
\Phi_{1}\left(x_{e+1}\right) \frac{\mathrm{d} \Phi_{1}\left(x_{e+1}\right)}{\mathrm{d} x} & \Phi_{1}\left(x_{e+1}\right) \frac{\mathrm{d} \Phi_{2}\left(x_{e+1}\right)}{\mathrm{d} x} & \Phi_{1}\left(x_{e+1}\right) \frac{\mathrm{d} \Phi_{3}\left(x_{e+1}\right)}{\mathrm{d} x} & \Phi_{1}\left(x_{e+1}\right) \frac{\mathrm{d} \Phi_{4}\left(x_{e+1}\right)}{\mathrm{d} x} \\
\Phi_{2}\left(x_{e+1}\right) \frac{\mathrm{d} \Phi_{1}\left(x_{e+1}\right)}{\mathrm{d} x} & \Phi_{2}\left(x_{e+1}\right) \frac{\mathrm{d} \Phi_{2}\left(x_{e+1}\right)}{\mathrm{d} x} & \Phi_{2}\left(x_{e+1}\right) \frac{\mathrm{d} \Phi_{3}\left(x_{e+1}\right)}{\mathrm{d} x} & \Phi_{2}\left(x_{e+1}\right) \frac{\mathrm{d} \Phi_{4}\left(x_{e+1}\right)}{\mathrm{d} x} \\
\Phi_{3}\left(x_{e+1}\right) \frac{\mathrm{d} \Phi_{1}\left(x_{e+1}\right)}{\mathrm{d} x} & \Phi_{3}\left(x_{e+1}\right) \frac{\mathrm{d} \Phi_{2}\left(x_{e+1}\right)}{\mathrm{d} x} & \Phi_{3}\left(x_{e+1}\right) \frac{\mathrm{d} \Phi_{3}\left(x_{e+1}\right)}{\mathrm{d} x} & \Phi_{3}\left(x_{e+1}\right) \frac{\mathrm{d} \Phi_{3}\left(x_{e+1}\right)}{\mathrm{d} x} \\
\Phi_{4}\left(x_{e+1}\right) \frac{\mathrm{d} \Phi_{1}\left(x_{e+1}\right)}{\mathrm{d} x} & \Phi_{4}\left(x_{e+1}\right) \frac{\mathrm{d} \Phi_{2}\left(x_{e+1}\right)}{\mathrm{d} x} & \Phi_{4}\left(x_{e+1}\right) \frac{\mathrm{d} \Phi_{3}\left(x_{e+1}\right)}{\mathrm{d} x} & \Phi_{4}\left(x_{e+1}\right) \frac{\mathrm{d} \Phi_{4}\left(x_{e+1}\right)}{\mathrm{d} x}
\end{array}\right]
$$

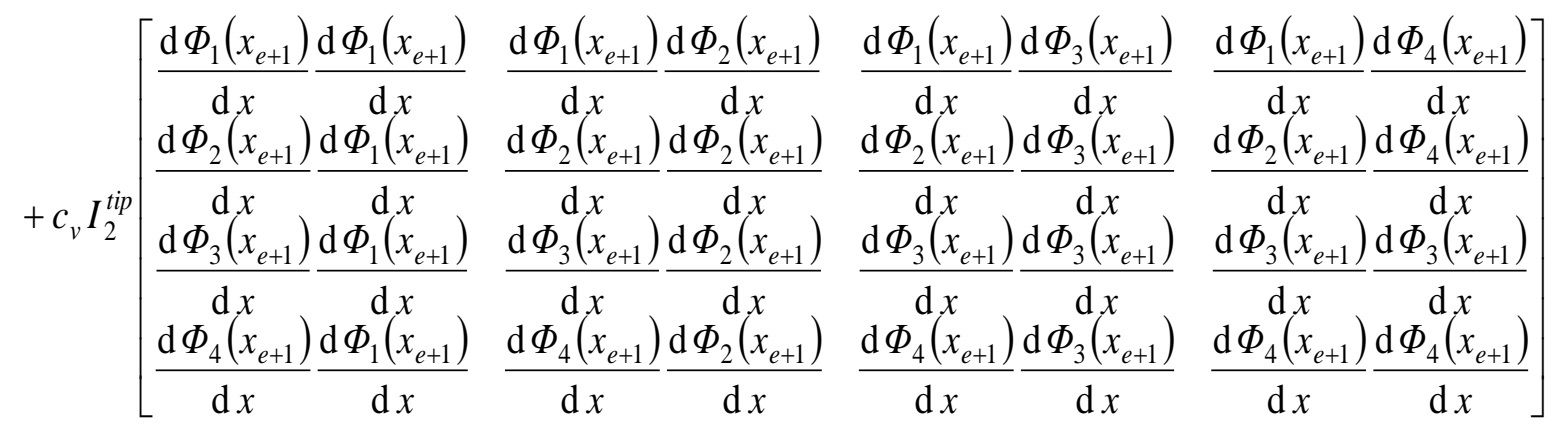

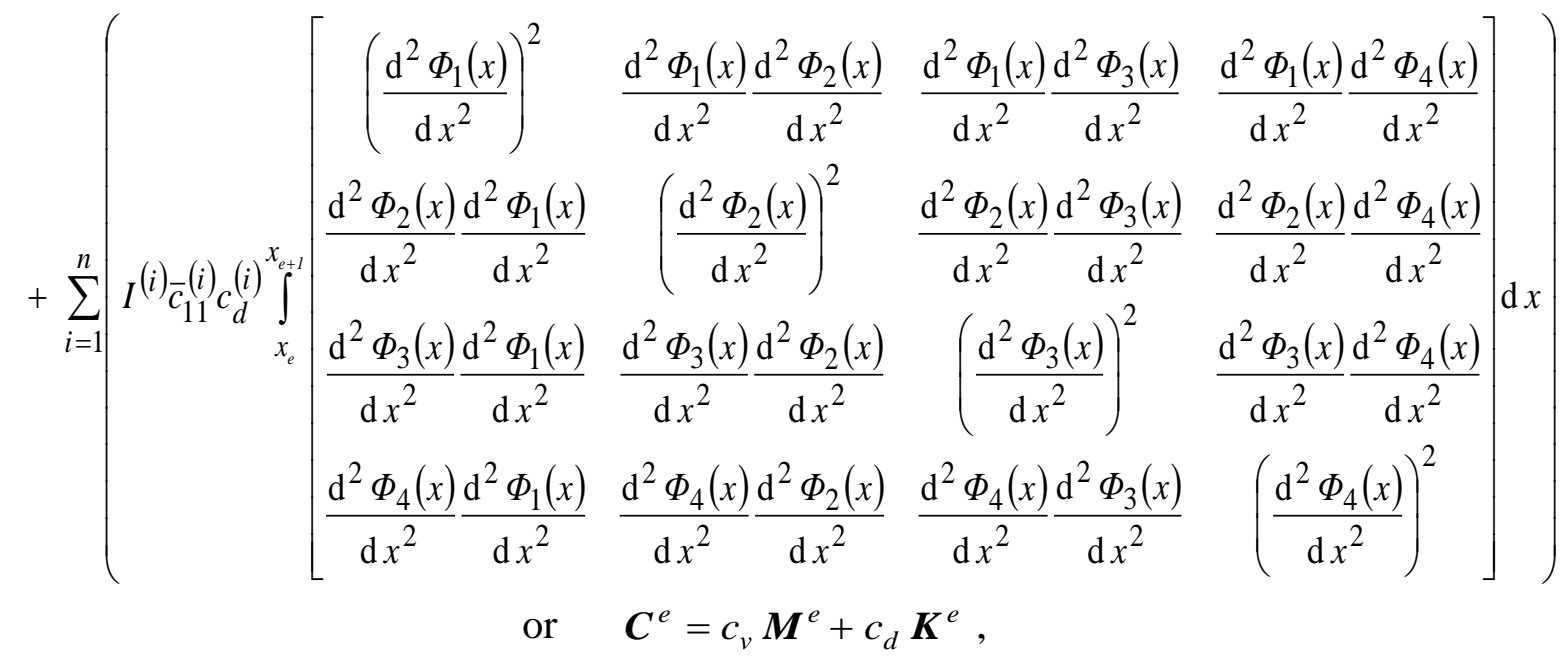

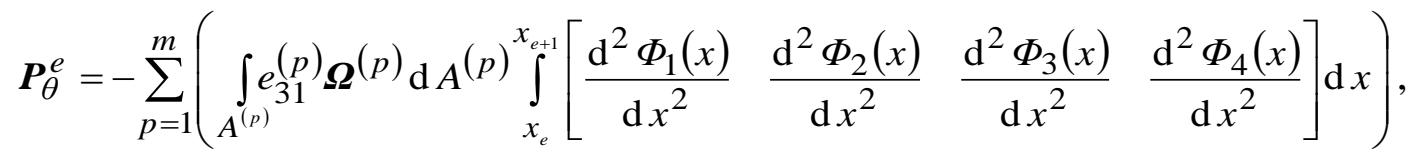

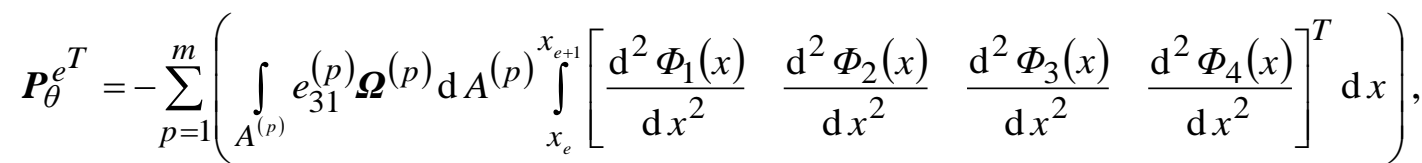

$$
\begin{aligned}
\boldsymbol{P}_{D}^{e} & =-\sum_{p=1}^{m}\left(\int_{A^{(p)}} L_{e} \varepsilon_{33}^{(p)} \boldsymbol{\Omega}^{(p)^{2}} \mathrm{~d} A^{(p)}\right), \quad \boldsymbol{F}^{e}=-\boldsymbol{Q}^{e} \ddot{\boldsymbol{w}}_{\text {base }}(t), \\
\boldsymbol{Q}^{e}= & \sum_{i=1}^{n}\left(\rho^{(i)} A^{(i)} \int_{x_{e}}^{x_{e+1}}\left[\begin{array}{llll}
\Phi_{1}(x) & \Phi_{2}(x) & \Phi_{3}(x) & \left.\Phi_{4}(x)\right]^{T} \mathrm{~d} x
\end{array}\right)\right. \\
& +I_{0}^{t i p} x_{c}\left[\begin{array}{llll}
\frac{\mathrm{d} \Phi_{1}\left(x_{e+1}\right)}{\mathrm{d} x} & \frac{\mathrm{d} \Phi_{2}\left(x_{e+1}\right)}{\mathrm{d} x} & \frac{\mathrm{d} \Phi_{3}\left(x_{e+1}\right)}{\mathrm{d} x} & \frac{\mathrm{d} \Phi_{4}\left(x_{e+1}\right)}{\mathrm{d} x}
\end{array}\right]^{T} \\
& +I_{0}^{t i p}\left[\begin{array}{llll}
\Phi_{1}\left(x_{e+1}\right) & \Phi_{2}\left(x_{e+1}\right) & \Phi_{3}\left(x_{e+1}\right) & \Phi_{4}\left(x_{e+1}\right)
\end{array}\right]^{T} .
\end{aligned}
$$


where $\boldsymbol{M}^{e}$ is the local mass matrix and $\boldsymbol{K}^{e}$ is the local stiffness matrix. Parameters $c_{v}$ and $c_{d}$ are the total Rayleigh damping coefficient of the structure, respectively. Other parameters $\boldsymbol{P}_{\theta}$, $\boldsymbol{P}_{D}$, and $\boldsymbol{F}$ indicate local electromechanical coupling matrices, local capacitance matrices, and local mechanical forces, respectively. Moreover, variable $\boldsymbol{i}_{p}$ is the local current output, $\boldsymbol{u}$ is the local mechanical coordinate, and $v$ is the local voltage output. Note that the effects of the rotary inertia of the proof mass and offset parameters are taken into account where previous major published works have ignored this case.

\subsection{Global matrices of electromechanical dynamic equation}

The global matrix forms of the structure with proof mass offset can be formulated using the generalised dynamic equations for each element of the structure to give,

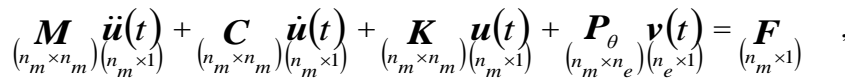

$$
\begin{aligned}
& \underset{\left(n_{e} \times n_{m}\right)}{\boldsymbol{P}_{\left(n_{m}\right.}^{T}} \underset{\left(n_{1}\right)}{\dot{\boldsymbol{u}}(t)}+\underset{\left(n_{e} \times n_{e}\right)}{\boldsymbol{P}_{\boldsymbol{b}}} \underset{\left(n_{e} \times 1\right)}{\dot{\boldsymbol{v}}(t)}=\underset{\left(n_{e} \times 1\right)}{\boldsymbol{i}_{p}(t)}
\end{aligned}
$$

Note that Eq. (24) consists of mechanical and electrical forms corresponding with the global matrices with the scripts $n_{m}$ and $n_{e}$, respectively. Here, the mechanical matrices correspond with the mechanical degrees of freedom of the structure for each node whereas electrical matrices correspond with electrical degrees of freedom for each element.

\subsection{Solution techniques using the orthonormalised global scalar forms}

The solution form of Eq. (24) can be formulated in terms of the normalised modal vector and time-dependent displacement generalised coordinate as,

$$
\boldsymbol{u}(t)=\boldsymbol{\varphi}_{1} \boldsymbol{a}_{1}(t)+\boldsymbol{\varphi}_{2} \boldsymbol{a}_{2}(t)+\ldots+\boldsymbol{\varphi}_{m-1} \boldsymbol{a}_{m-1}(t)+\boldsymbol{\varphi}_{m} \boldsymbol{a}_{m}(t)=\boldsymbol{\varphi} \boldsymbol{a}(t) .
$$

Since parameter $\varphi=\left[\begin{array}{llll}\varphi_{1} & \varphi_{2} & \ldots & \varphi_{m}\end{array}\right]$ is assumed to be a normalised modal matrix, the condition must meet the orthonormality relation with $\boldsymbol{\varphi}^{\mathrm{T}} \boldsymbol{M} \boldsymbol{\varphi}=1$. Let $\boldsymbol{\varphi}=c_{n} \boldsymbol{U}$ and parameter $c_{n}$ is the unknown arbitrary constant for each eigenvector while $\boldsymbol{U}$ is the known value of each eigenvector for each particular degree of freedom or eigenvalue. Therefore, it can be formulated as $1=c_{n}^{2} \boldsymbol{U}^{\mathrm{T}} \boldsymbol{M} \boldsymbol{U}$ such that $c_{n}=1 /\left(\boldsymbol{U}^{\mathrm{T}} \boldsymbol{M} \boldsymbol{U}\right)^{1 / 2}$. Finally, the normalised eigenvector or modal matrix can simply be formulated as $\boldsymbol{\varphi}=\boldsymbol{U} /\left(\boldsymbol{U}^{\mathrm{T}} \boldsymbol{M U}\right)^{1 / 2}$. 
Equation (24) can be further formulated by substituting Eq. (25) and premultiplying the result by $\boldsymbol{\varphi}^{T}$. The result of which can simply be formulated as,

$$
\begin{gathered}
\varphi^{T} M \varphi \ddot{a}(t)+\varphi^{T} C \varphi \dot{a}(t)+\varphi^{T} K \varphi a(t)+\varphi^{T} P_{\theta} v(t)=\varphi^{T} F \\
P_{\theta}^{T} \varphi \dot{a}(t)+P_{D} \dot{v}(t)=i_{p}(t)
\end{gathered}
$$

where orthonormalised parameters from Eq. (26) can be stated as,

$$
\begin{gathered}
\boldsymbol{\varphi}^{\boldsymbol{T}} \boldsymbol{M} \boldsymbol{\varphi}=\boldsymbol{I}, \quad \boldsymbol{\varphi}^{\boldsymbol{T}} \boldsymbol{K} \boldsymbol{\varphi}=\boldsymbol{\omega}^{2}, \boldsymbol{\varphi}^{\boldsymbol{T}} \boldsymbol{C} \boldsymbol{\varphi}=c_{v}\left(\boldsymbol{\varphi}^{T} \boldsymbol{M} \boldsymbol{\varphi}\right)+c_{d}\left(\boldsymbol{\varphi}^{T} \boldsymbol{K} \boldsymbol{\varphi}\right)=c_{v} \boldsymbol{I}+c_{d} \boldsymbol{\omega}^{2}=2 \zeta \boldsymbol{\omega}, \\
\hat{\boldsymbol{P}}_{\boldsymbol{\theta}}=\boldsymbol{\varphi}^{T} \boldsymbol{P}_{\theta}, \hat{\boldsymbol{P}}_{\theta}^{T}=\boldsymbol{P}_{\theta}^{T} \boldsymbol{\varphi}, \quad \hat{\boldsymbol{Q}}=\boldsymbol{\varphi}^{T} \boldsymbol{Q} .
\end{gathered}
$$

It should be noted that the first part of Eq. (27) represents the orthornormality property of the mechanical dynamic equations that show diagonal matrices. For this case, equation (26) can be simplified as,

$$
\begin{gathered}
\ddot{\boldsymbol{a}}(\boldsymbol{t})+2 \zeta \omega \dot{a}(t)+\omega^{2} a(t)+\hat{\boldsymbol{P}}_{\theta} v(t)=-\hat{Q}_{\boldsymbol{w}_{\text {base }}}(t), \\
\hat{\boldsymbol{P}}_{\theta}^{T} \dot{\boldsymbol{a}}(t)+\boldsymbol{P}_{D} \dot{\boldsymbol{v}}(t)=\boldsymbol{i}_{p}(t) .
\end{gathered}
$$

Global scalar form of the electromechanical dynamic equations can be further formulated using Eq. (28) in order to obtain the series form of the multimode FRFs. In this case, the first discretised electromechanical piezoelectric dynamic equation can be formulated in terms of the multi degree of freedom (multimode) system $r=1,2,3, \ldots, N D O F$ and the number of normalised piezoelectric elements $s=1,2,3, \ldots ., N E L P$ as,

$$
\begin{gathered}
\ddot{a}_{1}(t)+2 \zeta_{1} \omega_{1} \dot{a}_{1}(t)+\omega_{1}{ }^{2} a_{1}(t)+\hat{P}_{11} v_{1}(t)+\hat{P}_{12} v_{2}(t)+\cdots+\hat{P}_{1 s} v_{s}(t)=-\hat{Q}_{1} \ddot{w}_{\text {base }}(t), \\
\ddot{a}_{2}(t)+2 \zeta_{2} \omega_{2} \dot{a}_{2}(t)+\omega_{2}{ }^{2} a_{2}(t)+\hat{P}_{21} v_{1}(t)+\hat{P}_{22} v_{2}(t)+\cdots+\hat{P}_{2 s} v_{s}(t)=-\hat{Q}_{2} \ddot{w}_{\text {base }}(t), \\
\cdot \\
\cdot \\
\ddot{a}_{r}(t)+2 \zeta_{r} \omega_{r} \dot{a}_{r}(t)+\omega_{r}{ }^{2} a_{r}(t)+\sum_{s=1}^{N E L P} \hat{P}_{r s} v_{s}(t)=-\hat{Q}_{r} \ddot{w}_{\text {base }}(t), \quad r=1,2, \ldots, N D O F .
\end{gathered}
$$

The second form of the discretised electromechanical piezoelectric dynamic equation can be formulated as,

$$
\begin{aligned}
& \hat{P}_{11} \dot{a}_{1}(t)+\hat{P}_{12} \dot{a}_{2}(t)+\cdots+\hat{P}_{s r} \dot{a}_{r}(t)+P_{D 1} \dot{v}_{1}(t)=i_{P 1}(t) \\
& \hat{P}_{21} \dot{a}_{1}(t)+\hat{P}_{22} \dot{a}_{2}(t)+\cdots+\hat{P}_{s r} \dot{a}_{r}(t)+P_{D 2} \dot{v}_{2}(t)=i_{P 2}(t)
\end{aligned}
$$




$$
\sum_{r=1}^{N D O F} \hat{P}_{s r} \dot{a}_{r}(t)+P_{D s} \dot{v}_{s}(t)=i_{P s}(t), s=1,2,3, \ldots, N E L P
$$

The internal parallel connection in terms of the electrical discretised elements using Kirchhoff's voltage law (KVL) and Kirchhoff's current law (KCL) must be formulated in the scalar form as,

$$
v_{1}(t)=v_{2}(t)=\ldots .=v_{s}(t)=v(t) \quad, i_{P 1}(t)+i_{P 2}(t)+\ldots .+i_{P s}(t)=i_{P}(t) .
$$

Voltage output related to the external load resistance can be formulated as,

$$
v(t)=i_{P}(t) R_{\text {load }} .
$$

Equation (30) can be reformulated after applying the second part of Eq. (31) to give,

$$
\sum_{r=1}^{N D O F} \sum_{s=1}^{N E L P} \hat{P}_{s r} \dot{a}_{r}(t)+\sum_{s=1}^{N E L P} P_{D s} \dot{v}_{s}(t)=i_{P}(t)
$$

In this stage, after applying mathematical derivations using Eqs. (29)-(33), the multimode FRFs of the distributed piezoelectric unimorph can be formulated. Employing the first part of Eqs. (31) and (32) into Eqs. (29) and (33), respectively, the result of which can be further solved using Laplace transforms giving the result in matrix form. The first voltage multimode FRFs can be formulated to give,

$$
\frac{v(\mathrm{j} \omega)}{-\omega^{2} w_{\text {base }} e^{\mathrm{j} \omega t}}=\frac{\sum_{r=1}^{N D O F} \frac{\hat{Q}_{r} \mathrm{j} \omega \sum_{s=1}^{N E L P} \hat{P}_{s r}}{\omega_{r}{ }^{2}-\omega^{2}+\mathrm{j} 2 \zeta_{r} \omega_{r} \omega}}{\sum_{s=1}^{N E L P} \mathrm{j} \omega P_{D s}-\frac{1}{R_{\text {load }}}-\sum_{r=1}^{N D O F} \frac{\mathrm{j} \omega \sum_{s=1}^{N E L P} \hat{P}_{s r} \sum_{s=1}^{N E L P} \hat{P}_{r s}}{\omega_{r}{ }^{2}-\omega^{2}+\mathrm{j} 2 \zeta_{r} \omega_{r} \omega}}
$$

The multimode FRF of the electric current output related to the input base transverse acceleration can be stated as,

$$
\frac{i_{p}(\mathrm{j} \omega)}{-\omega^{2} w_{\text {base }} e^{\mathrm{j} \omega t}}=\frac{\frac{1}{R_{\text {load }}} \sum_{r=1}^{N D O F} \frac{\hat{Q}_{r} \mathrm{j} \omega \sum_{s=1}^{N E L P} \hat{P}_{s r}}{\omega_{r}^{2}-\omega^{2}+\mathrm{j} 2 \zeta_{r} \omega_{r} \omega}}{\sum_{s=1}^{N E L P} \mathrm{j} \omega P_{D s}-\frac{1}{R_{\text {load }}}-\sum_{r=1}^{N D O F} \frac{\mathrm{j} \omega \sum_{s=1}^{N E L P} \hat{P}_{s r} \sum_{s=1}^{N E L P} \hat{P}_{r s}}{\omega_{r}^{2}-\omega^{2}+\mathrm{j} 2 \zeta_{r} \omega_{r} \omega}} .
$$

The power harvesting multimode FRF related to the input transverse acceleration can be formulated as, 


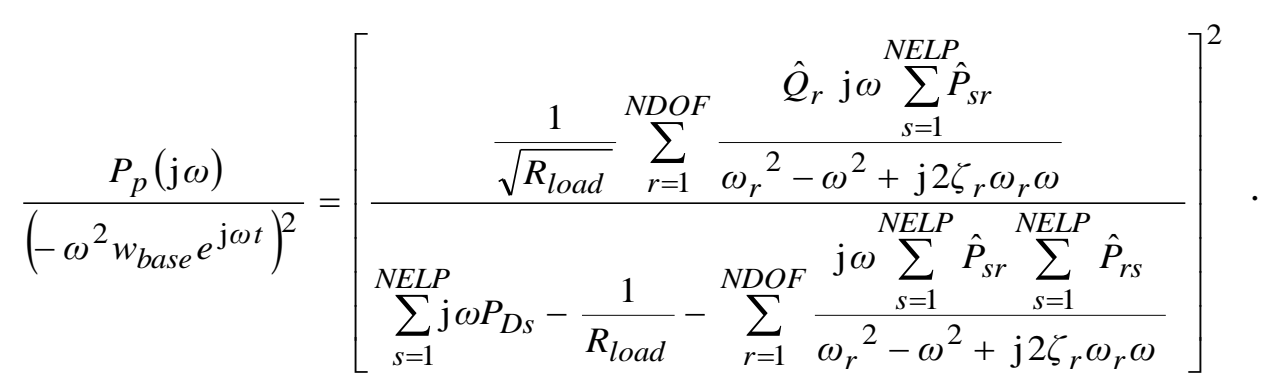

The optimal load resistance can be formulated by differentiating Eq. (36) with respect to load resistance and the differentiable power function can be set to zero to give,

$$
R_{\text {load }}^{\text {opt }}=\frac{\sqrt{X(\omega)^{2}+Y(\omega)^{2}}}{X(\omega)^{2}+Y(\omega)^{2}}
$$

where

$$
X(\omega)=\sum_{s=1}^{N E L P} \omega P_{D s}-\sum_{r=1}^{N D O F} \frac{\omega \sum_{s=1}^{N E L P} \hat{P}_{s r} \sum_{s=1}^{N E L P} \hat{P}_{r s}\left(\omega_{r}{ }^{2}-\omega^{2}\right)}{\left(\omega_{r}{ }^{2}-\omega^{2}\right)^{2}+\left(2 \zeta_{r} \omega_{r} \omega\right)^{2}}, Y(\omega)=\sum_{r=1}^{N D O F} \frac{\omega \sum_{s=1}^{N E L P} \hat{P}_{s r} \sum_{s=1}^{N E L P} \hat{P}_{r s}\left(2 \zeta_{r} \omega_{r} \omega\right)}{\left(\omega_{r}{ }^{2}-\omega^{2}\right)^{2}+\left(2 \zeta_{r} \omega_{r} \omega\right)^{2}} .
$$

It should be noted the optimal multimode FRF of power harvesting can be formulated by substituting back the optimal load resistance in Eq. (36). Moreover, the multimode FRF representing the transverse displacement relative to the input transverse acceleration can be obtained as,

$$
\frac{a_{r}(\mathrm{j} \omega)}{-\omega^{2} w_{\text {base }} e^{\mathrm{j} \omega t}}=-\frac{1}{\omega_{r}^{2}-\omega^{2}+\mathrm{j} 2 \zeta_{r} \omega_{r} \omega}\left(\frac{\left(\sum_{s=1}^{N E L P} \mathrm{j} \omega P_{D s}-\frac{1}{R_{\text {load }}}\right) \hat{Q}_{r}}{\sum_{s=1}^{N E L P} \mathrm{j} \omega P_{D s}-\frac{1}{R_{\text {load }}}-\sum_{r=1}^{N D O F} \frac{\mathrm{j} \omega \sum_{s=1}^{N E L P} \hat{P}_{s r} \sum_{s=1}^{N E L P} \hat{P}_{r s}}{\omega_{r}^{2}-\omega^{2}+\mathrm{j} 2 \zeta_{r} \omega_{r} \omega}}\right) .
$$

In terms of Eqs. (4) and (25), the characteristic transverse motion of the unimorph beam can be reformulated to give,

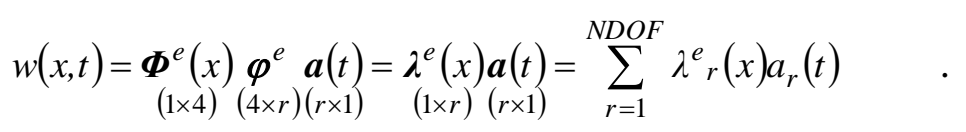

The FRF multimode relative transverse displacement related to the input base acceleration at any position along the unimorph beam ( $x$ ) can be formulated using Eqs. (39) and (40) as,

$$
\frac{w(x, \mathrm{j} \omega)}{-\omega^{2} w_{\text {base }} e^{\mathrm{j} \omega t}}=-\sum_{r=1}^{N D O F}\left[\frac{\lambda_{r}^{e}(x)}{\omega_{r}^{2}-\omega^{2}+\mathrm{j} 2 \zeta_{r} \omega_{r} \omega}\left(\frac{\left(\sum_{s=1}^{N E L P} \mathrm{j} \omega P_{D s}-\frac{1}{R_{\text {load }}}\right) \hat{Q}_{r}}{\sum_{s=1}^{N E L P} \mathrm{j} \omega P_{D s}-\frac{1}{R_{\text {load }}}-\sum_{r=1}^{N D O F} \frac{\mathrm{j} \omega \sum_{s=1}^{N E L P} \hat{P}_{s r} \sum_{s=1}^{N E L P} \hat{P}_{r s}}{\omega_{r}^{2}-\omega^{2}+\mathrm{j} 2 \zeta_{r} \omega_{r} \omega}}\right]\right)
$$


The absolute transverse displacement and velocity FRFs can be also be formulated as,

$$
\begin{aligned}
& \frac{w_{a b s}(x, \mathrm{j} \omega)}{-\omega^{2} w_{b a s e} e^{\mathrm{j} \omega t}}=-\frac{1}{\omega^{2}}+\frac{w(x, \mathrm{j} \omega)}{-\omega^{2} w_{b a s e} e^{\mathrm{j} \omega t}}, \\
& \frac{\dot{w}_{a b s}(x, \mathrm{j} \omega)}{-\omega^{2} w_{b a s e} e^{\mathrm{j} \omega t}}=\frac{1}{\mathrm{j} \omega}+\mathrm{j} \omega \frac{w(x, \mathrm{j} \omega)}{-\omega^{2} w_{\text {base }} e^{\mathrm{j} \omega t}} .
\end{aligned}
$$

The transverse displacement response of the proof mass offset can be formulated over the interval $L \leq x \leq L+L_{t i p}$ as,

$$
\begin{aligned}
& \boldsymbol{w}\left(L+L_{t i p}, t\right)=\boldsymbol{w}(L, t)+L_{t i p} \frac{\mathrm{d} \boldsymbol{w}(L, t)}{\mathrm{d} x}=\left(\underset{(1 \times 4)}{\boldsymbol{\Phi}^{e}(L)+L_{t i p}} \frac{\mathrm{d} \boldsymbol{\Phi}^{e}(L)}{\underset{(1 \times 4)}{\mathrm{d} x}}\right) \underset{(4 \times r)(r \times 1)}{\boldsymbol{\varphi}^{e}} \boldsymbol{a}(t) \\
& =\lambda^{e} \underset{(1 \times r)}{\left(L+L_{t i p}\right) \underset{(r \times 1)}{a}(t)}=\sum_{r=1}^{N D O F} \lambda^{e} r\left(L+L_{t i p}\right) a_{r}(t)
\end{aligned}
$$

The multimode transverse displacement FRFs for the proof mass offset can also be formulated in terms of Eqs. (39) and (43) to give,

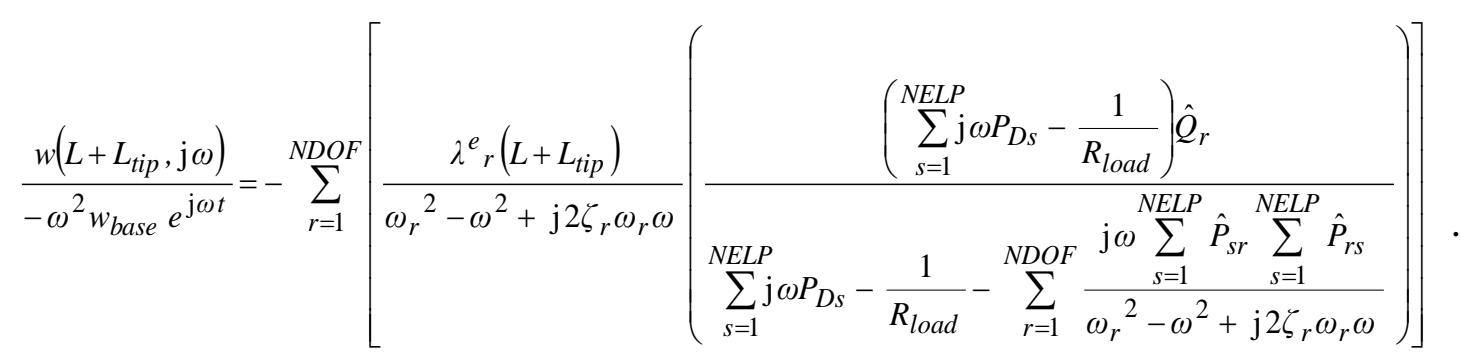

The absolute transverse displacement and velocity FRFs at any position along the proof mass offset can be formulated as,

$$
\begin{aligned}
& \frac{w_{a b s}\left(L+L_{t i p}, \mathrm{j} \omega\right)}{-\omega^{2} w_{b a s e} e^{\mathrm{j} \omega t}}=-\frac{1}{\omega^{2}}+\frac{w\left(L+L_{t i p}, \mathrm{j} \omega\right)}{-\omega^{2} w_{b a s e} e^{\mathrm{j} \omega t}} \\
& \frac{\dot{w}_{a b s}\left(L+L_{t i p}, \mathrm{j} \omega\right)}{-\omega^{2} w_{b a s e} e^{\mathrm{j} \omega t}}=\frac{1}{\mathrm{j} \omega}+\mathrm{j} \omega \frac{w\left(L+L_{t i p}, \mathrm{j} \omega\right)}{-\omega^{2} w_{b a s e} e^{\mathrm{j} \omega t}} .
\end{aligned}
$$

\section{Formulations of Electromechanical Closed-Form Boundary Value Method}

This section focuses on the analytical method of electromechanical closed-form boundary value method for formulating the system responses of the unimorph beam with arbitrary proof mass offset using the Hamiltonian principle which can be formulated as,

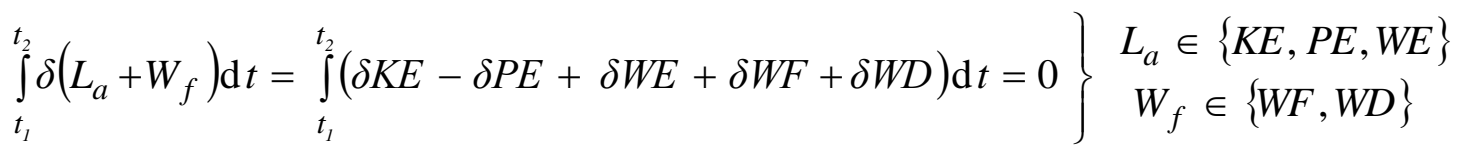


Note that each term of Eq. (15) can be formulated in Eqs. (47)-(51). With these parameters, the similar forms can also be found in numerical methods as shown in Eqs. (14)-(18). The only difference between the parameters shown in these equations is that the local element length of structure $x_{e+1}$ for the numerical method as shown in Fig. (2b) can be transformed into $L$ for analytical method.

The kinetic energy of the structure can be reformulated as,

$$
\begin{aligned}
K E= & \frac{1}{2} \int_{0}^{L} \int_{A^{(1)}} \rho^{(1)} \dot{w}(x, t)^{2} \mathrm{~d} A^{(1)} \mathrm{d} x+\frac{1}{2} \int_{0}^{L} \int_{A^{(2)}} \rho^{(2)} \dot{w}(x, t)^{2} \mathrm{~d} A^{(2)} \mathrm{d} x \\
& +x_{c} I_{0}^{t i p} \dot{w}(L, t) \dot{\theta}(L, t)+\frac{1}{2} I_{0}^{t i p} \dot{w}(L, t)^{2}+\frac{1}{2} I_{2}^{t i p} \dot{\theta}(L, t)^{2} .
\end{aligned}
$$

Note that detail of the mathematical equations for the dynamical beam structure and proof mass offset as shown in the kinetic energy can be found in [27]. The potential energy due to the stress-strain-electric-damping stress relation for the unimorph layers can be formulated as,

$$
P E=\frac{1}{2} \int_{0}^{L} \int_{A^{(1)}} S_{1}^{(1)} T_{1}^{(1)} \mathrm{d} A^{(1)} \mathrm{d} x+\frac{1}{2} \int_{0}^{L} \int_{A^{(2)}} S_{1}^{(2)} T_{1}^{(2)} \mathrm{d} A^{(2)} \mathrm{d} x .
$$

The electrical energy term for the piezoelectric element can be formulated as,

$$
W E=\frac{1}{2} \int_{0}^{L} \int_{A^{(2)}} E_{3} D_{3} \mathrm{~d} A^{(2)} \mathrm{d} x
$$

The non-conservative work on the system due to the input base excitation and electrical charge output can be stated as,

$$
\begin{aligned}
W F=- & \int_{0}^{L} \int_{A^{(1)}} \rho^{(1)} w(x, t) \mathrm{d} A^{(1)} \mathrm{d} x \ddot{w}_{\text {base }}(t)-\int_{0}^{L} \int_{A^{(2)}} \rho^{(2)} w(x, t) \mathrm{d} A^{(2)} \mathrm{d} x \ddot{w}_{\text {base }}(t) \\
& -x_{c} I_{0}^{t i p} \theta(L, t) \ddot{w}_{\text {base }}(t)-I_{0}^{t i p} w(L, t) \ddot{w}_{\text {base }}(t)+q^{(e)}(t) v^{(e)}(t) .
\end{aligned}
$$

The power dissipated by air friction on the unimorph and proof mass offset using Rayleigh's dissipation function can be stated as,

$$
\begin{aligned}
P D= & -\frac{1}{2} \int_{0}^{L} \int_{A^{(1)}} c_{v} \rho^{(1)} \dot{w}(x, t)^{2} \mathrm{~d} A^{(1)} \mathrm{d} x-\frac{1}{2} \int_{0}^{L} \int_{A^{(2)}} c_{v} \rho^{(2)} \dot{w}(x, t)^{2} \mathrm{~d} A^{(2)} \mathrm{d} x \\
& -x_{c} c_{v} I_{0}^{t i p} \dot{\theta}(L, t) \dot{w}(L, t)-\frac{1}{2} c_{v} I_{0}^{t i p} \dot{w}(L, t)^{2}-\frac{1}{2} c_{v} I_{2}^{t i p} \dot{\theta}(L, t)^{2} .
\end{aligned}
$$

or Eq. (51) can be modified into the work done due to air friction using the relation $\delta W D=\frac{\partial P D}{\partial \dot{y}} \delta y \forall y \in\left\{w(x, t), \frac{\partial w}{\partial x}(L, t), w(L, t)\right\}$ to give,

$$
\delta W D=-\int_{0}^{L} \int_{A^{(1)}} c_{v} \rho^{(1)} \dot{w}(x, t) \mathrm{d} A^{(1)} \mathrm{d} x \delta w(x, t)-\int_{0}^{L} \int_{A^{(2)}} c_{v} \rho^{(2)} \dot{w}(x, t) \mathrm{d} A^{(2)} \mathrm{d} x \delta w(x, t)-x_{c} c_{v} I_{0}^{t i p} \dot{\theta}(L, t) \delta w(L, t)
$$




$$
-x_{c} c_{v} I_{0}^{t i p} \dot{w}(L, t) \delta \theta(L, t)-c_{v} I_{0}^{t i p} \dot{w}(L, t) \delta w(L, t)-c_{v} I_{2}^{t i p} \dot{\theta}(L, t) \delta \theta(L, t) .
$$

Detail of derivation of functional form for damping relations can be seen in the next stage. The functional forms $L_{a}$ and $W_{f}$ from Hamiltonian's principle shows characteristic virtual multi-variable in relation to the variational principle. The functional forms $L_{a}$ and $W_{f}$ can be stated as,

$$
\begin{gathered}
L_{a}=L_{a}\left(\dot{w}(x, t), \dot{w}(L, t), \frac{\partial \dot{w}(L, t)}{\partial x}, \frac{\partial^{2} w(x, t)}{\partial x^{2}}, E(z, t)\right), \\
W_{f}=W_{f}\left(w(x, t), \frac{\partial w(L, t)}{\partial x}, w(L, t), v(t)\right) .
\end{gathered}
$$

Equations (53) and (54) can be further formulated using the total differential equations as,

$$
\delta L_{a}=\overbrace{\frac{\partial L_{a}}{\partial \dot{w}(x, t)} \delta \dot{w}(x, t)+\frac{\partial L_{a}}{\partial \dot{w}(L, t)} \delta \dot{w}(L, t)+\frac{\partial L_{a}}{\partial\left(\frac{\partial \dot{w}}{\partial x}(L, t)\right)} \delta\left(\frac{\partial \dot{w}}{\partial x}(L, t)\right)}^{\begin{array}{c}
\text { Virtual kinetic energy based on the generalised } \\
\text { velocities of structure and tip mass offset }
\end{array}}
$$

Virtual potential energy based on the generalised strain and the generalised strain rate due to internal damping

$+\overbrace{\frac{\partial L_{a}}{\partial\left(\frac{\partial^{2} w(x, t)}{\partial x^{2}}\right)} \delta\left(\frac{\partial^{2} w(x, t)}{\left.\partial x^{2}\right)}\right)}^{\text {based on the electric field }}$,

$$
\delta W_{f}=\overbrace{\frac{\partial P_{D}}{\partial \dot{w}(x, t)} \delta w(x, t)+\frac{\partial P_{D}}{\partial\left(\frac{\partial \dot{w}}{\partial x}(L, t)\right)} \delta\left(\frac{\partial w}{\partial x}(L, t)\right)+\frac{\partial P_{D}}{\partial \dot{w}(L)} \delta w(L, t)}^{\begin{array}{c}
\text { Virtual work based on the generalise d } \\
\text { displacement due to air damping }
\end{array}}
$$

Virtual work based on generalise d displacem ent of the structure and proof mass offset

$\overbrace{\frac{\partial W_{f}}{\partial w(x, t)} \delta w(x, t)+\frac{\partial W_{f}}{\partial\left(\frac{\partial w}{\partial x}(L, t)\right)} \delta\left(\frac{\partial w}{\partial x}(L, t)\right)+\frac{\partial W_{f}}{\partial w(L)} \delta w(L, t)}+\overbrace{\frac{\partial W_{f}}{\partial v(t)} \delta v(t)}$

Note that the first to third terms in Eq. (56) indicate the differential form of power - work relations due to air friction that can be proved. Let the functional form of power dissipation be $\delta P_{D}=Q_{f} \delta \dot{y} \forall \dot{y} \in\left\{\dot{w}(x, t), \frac{\partial \dot{w}}{\partial x}(L, t), \dot{w}(L, t)\right\}$ and let the variation of work done on the system be $\delta W_{f}=Q_{f} \delta y$, such that $f: W_{f} \rightarrow\{y\}$ gives the total differential form $\delta W_{f}=\frac{\partial P_{D}}{\partial \dot{y}} \delta y \forall y \in\left\{w(x, t), \frac{\partial w}{\partial x}(L, t), w(L, t)\right\}$ where $Q_{f}=\frac{\partial P_{D}}{\partial \dot{y}}$ is applied friction force on 
the system. To meet the Hamiltonian principle as shown in equation (46), $\delta W_{f}$ must be the dependent variable. Moreover, the contribution of damping stress due to internal friction in the elemental structure can be seen in Eq. (55) at the sixth term.

In terms of Eqs. (55) and (56), equation (46) can be further formulated using integrodifferential equations and extended using the variational principle in order to meet the continuous differentiable functions in the elemental structure including its boundary conditions in terms of virtual displacement, rotation and electrical voltage. After simplification, the reduced integro-differential equation of the electromechanical modal damped vibrational piezoelectric structure can be formulated as,

$$
\begin{aligned}
& \int_{t_{l}}^{t_{2}}\left[\int_{0}^{L}\left\{-I_{0} \ddot{w}(x, t)-I_{0} \ddot{w}_{\text {base }}(t)-c_{v} I_{0} \dot{w}(x, t)-c_{d} C_{s} \frac{\partial^{2}}{\partial x^{2}}\left(\frac{\partial^{2} \dot{w}(x, t)}{\partial x^{2}}\right)-C_{s} \frac{\partial^{2}}{\partial x^{2}}\left(\frac{\partial^{2} w(x, t)}{\partial x^{2}}\right)\right\} \delta w(x, t) d x\right. \\
& \quad-\left\{x_{c} I_{0}^{t i p} \frac{\partial \ddot{w}(L, t)}{\partial x}+I_{0}^{t i p} \ddot{w}(L, t)+x_{c} c_{v} I_{0}^{t i p} \frac{\partial \dot{w}(L, t)}{\partial x}+c_{v} I_{0}^{t i p} \dot{w}(L, t)+I_{0}^{t i p} \ddot{w}_{\text {base }}\right\} \delta w(L, t) \\
& +\left.\left(c_{d} C_{s} \frac{\partial}{\partial x}\left(\frac{\partial^{2} \dot{w}(x, t)}{\partial x^{2}}\right)+C_{s} \frac{\partial}{\partial x}\left(\frac{\partial^{2} w(x, t)}{\partial x^{2}}\right)\right) \delta w(x, t)\right|_{0} ^{L}-\left\{I_{2}^{t i p} \frac{\partial \ddot{w}(L, t)}{\partial x}+x_{c} I_{0}^{t i p} \ddot{w}_{\text {base }}+x_{c} I_{0}^{t i p} \ddot{w}(L, t)\right. \\
& \left.+c_{v} I_{2}^{t i p} \frac{\partial \dot{w}(L, t)}{\partial x}+x_{c} c_{v} I_{0}^{t i p} \dot{w}(L, t)\right\} \delta \frac{\partial w(L, t)}{\partial x}-\left.\mu v(t) \delta \frac{\partial w(x, t)}{\partial x}\right|_{0} ^{L}-\left(c_{d} C_{s} \frac{\partial^{2} \dot{w}(x, t)}{\partial x^{2}}+C_{s} \frac{\partial^{2} w(x, t)}{\left.\partial x^{2}\right)\left.\delta \frac{\partial w(x, t)}{\partial x}\right|_{0} ^{L}}\right. \\
& \left.-\left\{\int_{0}^{L}\left(\mu \frac{\partial^{2} w(x, t)}{\partial x^{2}}-C_{p} v(t)\right) \mathrm{d} x-q(t)\right\} \delta v(t)\right] \mathrm{d} t=0 .
\end{aligned}
$$

It is important to note here that equation (57) shows the complete equation with the effect of arbitrary proof mass offset and damping components. Parameters $I_{0}, I_{0}^{\text {tip }}, I_{2}^{\text {tip }}, C_{s}, C_{p}$ and $\mu$ can be found in Appendices A, B and C. Note that the reduced equation must meet the mathematical lemma of the variational method of duBois-Reymond's theorem for each virtual displacement field. The first constitutive electromechanical damped dynamic equation can be formulated as,

$$
\delta w(x, t): I_{0} \ddot{w}(x, t)+I_{0} \ddot{w}_{b a s e}(t)+c_{v} I_{0} \dot{w}(x, t)+c_{d} C_{s} \frac{\partial^{2}}{\partial x^{2}}\left(\frac{\partial^{2} \dot{w}(x, t)}{\partial x^{2}}\right)+C_{s} \frac{\partial^{2}}{\partial x^{2}}\left(\frac{\partial^{2} w(x, t)}{\partial x^{2}}\right)=0
$$

The second constitutive electromechanical dynamic equation can be formulated as,

$$
\delta v(t): \int_{0}^{L}\left(-\mu \frac{\partial^{2} w(x, t)}{\partial x^{2}}+C_{p} v(t)\right) \mathrm{d} x+q(t)=0 .
$$


The boundary conditions can also be reduced to give,

$$
\begin{gathered}
w(0, t)=0 \quad, \frac{\partial w(0, t)}{\partial x}=0, \\
\delta w(L, t): \quad x_{c} I_{0}^{t i p} \frac{\partial \ddot{w}(L, t)}{\partial x}+I_{0}^{t i p} \ddot{w}(L, t)+I_{0}^{t i p} \ddot{w}_{\text {base }}+x_{c} c_{v} I_{0}^{t i p} \frac{\partial \dot{w}(L, t)}{\partial x}+c_{v} I_{0}^{t i p} \dot{w}(L, t) \\
\quad-c_{d} C_{s} \frac{\partial}{\partial x}\left(\frac{\partial^{2} \dot{w}(L, t)}{\partial x^{2}}\right)-C_{s} \frac{\partial}{\partial x}\left(\frac{\partial^{2} w(L, t)}{\partial x^{2}}\right)=0, \\
\delta \frac{\partial w(L, t)}{\partial x}: \quad x_{c} I_{0}^{t i p} \ddot{w}(L, t)+x_{c} I_{0}^{t i p} \ddot{w}_{b a s e}+I_{2}^{t i p} \frac{\partial \ddot{w}(L, t)}{\partial x}+c_{v} I_{2}^{t i p} \frac{\partial \dot{w}(L, t)}{\partial x}+x_{c} c_{v} I_{0}^{t i p} \dot{w}(L, t) \\
+c_{d} C_{s} \frac{\partial^{2} \dot{w}(L, t)}{\partial x^{2}}+C_{s} \frac{\partial^{2} w(L, t)}{\partial x^{2}}+\mu v(t)=0 .
\end{gathered}
$$

Note that since the system is under base excitation as shown in Fig. 1, Eqs. (58)-(60) reduced from Eq. (57) described the unimorph smart structure with a proof mass offset operating under the dynamical motion where the mathematical expressions of the dynamical system was reduced due to the relative displacement $w(x, t)$ defined as the difference between absolute displacement $w_{a b s}(x, t)$ and base excitation $w_{b a s e}(t)$. Details of the kinematic equations can be found in [27]. It is clearly seen that damping effects due to air friction and internal friction on the system also contribute not only to the constitutive electromechanical damped dynamic equation, but also in the boundary conditions where most published papers either in the piezoelectric power harvester or other integrated piezoelectric applications have not included derivations of the damping effect and normally have added it into the final constitutive equations for simplicity as formulated into Rayleigh damping $c_{r q}=c_{v} M_{r q}+c_{d} K_{r q}$ or normalised Rayleigh damping form $c_{r q}=c_{v} \delta_{r q}+c_{d} \omega_{r}{ }^{2} \delta_{r q}=2 \zeta_{r} \omega_{r} \delta_{r q}$. Note that since the piezoelectric beam is vibrated on the air, the air damping coefficient occurs due to the kinetic energy from the velocity of the beam motion creating air particle friction whereas the strain-rate damping occurs due to the internal friction of the material during vibrational motion [30]. Note that the air damping coefficient is sometimes called the mass proportional damping coefficient whereas internal friction damping coefficient is sometimes known as the structural stiffness proportional damping coefficient, reflecting the Rayleigh damping coefficient [31]. As shown in the electromechanical damping derivations in the finite element modelling previously, the analytical techniques proposed here also associate with the Rayleigh damping derivations. 
The closed-form analytical method using the electromechanical dynamic equations associated with the boundary conditions can be further formulated using the convergent eigenfunction forms which can be formulated as,

$$
w(x, t)=\sum_{r=1}^{\infty} \hat{W}_{r}(x) w_{r}(t) .
$$

Equation (61) is sometimes called mode superposition which depends on the normalised mode shapes and generalised time dependent coordinates. Note that the normalised mode shape can be found in Appendix D. The new forms of equations (58)-(60) can be expressed using the normalised eigenfunction series. In terms of Eq. (58), the first electromechanical equation can be reformulated using (61) and the results can be multiplied with $\hat{W}_{q}(x)$ giving,

$$
\begin{gathered}
\int_{0}^{L} I_{0} \hat{W}_{r}(x) \hat{W}_{q}(x) \ddot{w}_{r}(t) \mathrm{d} x+\int_{0}^{L} c_{v} I_{0} \hat{W}_{r}(x) \hat{W}_{q}(x) \dot{w}_{r}(t)+\int_{0}^{L} c_{d} C_{s} \frac{\mathrm{d}^{2}}{\mathrm{~d} x^{2}}\left(\frac{\mathrm{d}^{2} \hat{W}_{r}(x)}{\mathrm{d} x^{2}}\right) \hat{W}_{q}(x) \dot{w}_{r}(t) \mathrm{d} x \\
+\int_{0}^{L} C_{s} \frac{\mathrm{d}^{2}}{\mathrm{~d} x^{2}}\left(\frac{\mathrm{d}^{2} \hat{W}_{r}(x)}{\mathrm{d} x^{2}}\right) \hat{W}_{q}(x) w_{r}(t) \mathrm{d} x+\int_{0}^{L} I_{0} \hat{W}_{q}(x) \ddot{w}_{\text {base }}(t) \mathrm{d} x=0 .
\end{gathered}
$$

The second electromechanical dynamic equation from Eq. (59) can be further formulated by applying (61) and differentiating it with respect to time to give,

$$
-\sum_{r=1}^{\infty} \int_{0}^{L} \mu \frac{\mathrm{d}^{2} \hat{W}_{r}(x)}{\mathrm{d} x^{2}} \mathrm{~d} x \dot{w}_{r}(t)+\int_{0}^{L} C_{p} \mathrm{~d} x \dot{v}(t)+i_{P}(t)=0 .
$$

The boundary conditions from Eq. (60) can also be further formulated by substituting Eq. (61) as,

$$
\begin{gathered}
\hat{W}_{r}(0)=0 \quad, \quad \frac{\mathrm{d} \hat{W}_{r}(0)}{\mathrm{d} x}=0, \\
x_{c} I_{0}^{t i p} \frac{\mathrm{d} \hat{W}_{r}(L)}{\mathrm{d} x} \ddot{w}(t)+I_{0}^{t i p} \hat{W}_{r}(L) \ddot{w}(t)+I_{0}^{t i p} \ddot{w}_{\text {base }}+x_{c} c_{v} I_{0}^{t i p} \frac{\mathrm{d} \hat{W}_{r}(L)}{\mathrm{d} x} \dot{w}(t)+c_{v} I_{0}^{t i p} \hat{W}_{r}(L) \dot{w}(t) \\
-c_{d} C_{s} \frac{\mathrm{d}}{\mathrm{d} x}\left(\frac{\mathrm{d}^{2} \hat{W}_{r}(L)}{\mathrm{d} x^{2}}\right) \dot{w}_{r}(t)-C_{s} \frac{\mathrm{d}}{\mathrm{d} x}\left(\frac{\mathrm{d}^{2} \hat{W}_{r}(L)}{\mathrm{d} x^{2}}\right) w_{r}(t)=0, \\
x_{c} I_{0}^{t i p} \hat{W}_{r}(L) \ddot{w}(t)+x_{c} I_{0}^{t i p} \ddot{w}_{b a s e}+I_{2}^{t i p} \frac{\mathrm{d} \hat{W}_{r}(L)}{\mathrm{d} x} \ddot{w}(t)+c_{v} I_{2}^{t i p} \frac{\mathrm{d} \hat{W}_{r}(L)}{\mathrm{d} x} \dot{w}(t)+c_{v} x_{c} I_{0}^{t i p} \hat{W}_{r}(L) \dot{w}(t) \\
+c_{d} C_{s} \frac{\mathrm{d}^{2} \hat{W}_{r}(L)}{\mathrm{d} x^{2}} \dot{w}(t)+C_{s} \frac{\mathrm{d}^{2} \hat{W}_{r}(L)}{\mathrm{d} x^{2}} w(t)+\mu v(t)=0 .
\end{gathered}
$$

In terms of orthogonality relation, the third and fourth terms of Eq. (62) needs to be further manipulated by using partial integration, the result of which can be further formulated by 
applying the boundary conditions from the first part in Eq. (64). The resulting coupled stiffness-damping differential equation can be written as,

$$
\begin{aligned}
& \int_{0}^{L} \frac{\mathrm{d}^{2}}{\mathrm{~d} x^{2}}\left(\frac{\mathrm{d}^{2} \hat{W}_{r}(x)}{\mathrm{d} x^{2}}\right) \hat{W}_{q}(x) \mathrm{d} x\left(C_{s} w_{r}(t)+c_{d} C_{s} \dot{w}_{r}(t)\right)=\frac{\mathrm{d}}{\mathrm{d} x}\left(\frac{\mathrm{d}^{2} \hat{W}_{r}(L)}{\mathrm{d} x^{2}}\right) \hat{W}_{q}(L)\left(C_{s} w_{r}(t)+c_{d} C_{s} \dot{w}_{r}(t)\right) \\
& -\frac{\mathrm{d}^{2} \hat{W}_{r}(L)}{\mathrm{d} x^{2}} \frac{\mathrm{d} \hat{W}_{q}(L)}{\mathrm{d} x}\left(C_{s} w_{r}(t)+c_{d} C_{s} \dot{w}_{r}(t)\right)+\int_{0}^{L} \frac{\mathrm{d}^{2} \hat{W}_{r}(x)}{\mathrm{d} x^{2}} \frac{\mathrm{d}^{2} \hat{W}_{q}(x)}{\mathrm{d} x^{2}} \mathrm{~d} x\left(C_{s} w_{r}(t)+c_{d} C_{s} \dot{w}_{r}(t)\right) .
\end{aligned}
$$

In terms of conditions implied in the second and third equations of Eq. (64), equation (65) can be formulated as,

$$
\begin{aligned}
& \quad \int_{0}^{L} \frac{\mathrm{d}^{2}}{\mathrm{~d} x^{2}}\left(\frac{\mathrm{d}^{2} \hat{W}_{r}(x)}{\mathrm{d} x^{2}}\right) \hat{W}_{q}(x) d x\left(C_{s} w_{r}(t)+c_{d} C_{s} \dot{w}_{r}(t)\right)=I_{0}^{t i p} \hat{W}_{q}(L) \ddot{w}_{\text {base }}+I_{0}^{t i p} \hat{W}_{r}(L) \hat{W}_{q}(L) \ddot{w}_{r}(t) \\
& +x_{c} I_{0}^{t i p} \frac{\mathrm{d} \hat{W}_{r}(L)}{\mathrm{d} x} \hat{W}_{q}(L) \ddot{w}_{r}+x_{c} I_{0}^{t i p} \hat{W}_{r}(L) \frac{\mathrm{d} \hat{W}_{q}(L)}{\mathrm{d} x} \ddot{w}_{r}+x_{c} I_{0}^{t i p} \frac{\mathrm{d} \hat{W}_{q}(L)}{\mathrm{d} x} \ddot{w}_{b a s e}+x_{c} c_{v} I_{0}^{t i p} \frac{\mathrm{d} \hat{W}_{r}(L)}{\mathrm{d} x} \hat{W}_{q}(L) \dot{w}(t) \\
& +c_{v} I_{0}^{t i p} \hat{W}_{r}(L) \hat{W}_{q}(L) \dot{w}(t)+c_{v} I_{2}^{t i p} \frac{\mathrm{d} \hat{W}_{r}(L)}{\mathrm{d} x} \frac{\mathrm{d} \hat{W}_{q}(L)}{\mathrm{d} x} \dot{w}(t)+x_{c} c_{v} I_{0}^{t i p} \hat{W}_{r}(L) \frac{\mathrm{d} \hat{W}_{q}(L)}{\mathrm{d} x} \dot{w}(t) \\
& +I_{2}^{t i p} \frac{\mathrm{d} \hat{W}_{r}(L)}{\mathrm{d} x} \frac{\mathrm{d} \hat{W}_{q}(L)}{\mathrm{d} x} \ddot{w}_{r}(t)+\mu \frac{\mathrm{d} \hat{W}_{q}(L)}{\mathrm{d} x} v(t)+\int_{0}^{L} \frac{\mathrm{d}^{2} \hat{W}_{r}(x)}{\mathrm{d} x^{2}} \frac{\mathrm{d}^{2} \hat{W}_{q}(x)}{\mathrm{d} x^{2}} \mathrm{~d} x\left(C_{s} w_{r}(t)+c_{d} C_{s} \dot{w}_{r}(t)\right) . \quad(66)
\end{aligned}
$$

Corresponding to Eq. (66), equation (62) can be reformulated to give,

$$
\begin{aligned}
& \int_{0}^{L} I_{0} \hat{W}_{r}(x) \hat{W}_{q}(x) \mathrm{d} x \ddot{w}_{r}(t)+I_{0}^{t i p} \hat{W}_{r}(L) \hat{W}_{q}(L) \ddot{w}_{r}(t)+x_{c} I_{0}^{t i p} \frac{\mathrm{d} \hat{W}_{r}(L)}{\mathrm{d} x} \hat{W}_{q}(L) \ddot{w}_{r}(t) \\
& +x_{c} I_{0}^{t i p} \hat{W}_{r}(L) \frac{\mathrm{d} \hat{W}_{q}(L)}{\mathrm{d} x} \ddot{w}_{r}(t)+I_{2}^{t i p} \frac{\mathrm{d} \hat{W}_{r}(L)}{\mathrm{d} x} \frac{\mathrm{d} \hat{W}_{q}(L)}{\mathrm{d} x} \ddot{w}_{r}(t)+\int_{0}^{L} c_{v} I_{0} \hat{W}_{r}(x) \hat{W}_{q}(x) \dot{w}_{r}(t) \\
& +c_{v} I_{0}^{t i p} \hat{W}_{r}(L) \hat{W}_{q}(L) \dot{w}(t)+x_{c} c_{v} I_{0}^{t i p} \frac{\mathrm{d} \hat{W}_{r}(L)}{\mathrm{d} x} \hat{W}_{q}(L) \dot{w}(t)+x_{c} c_{v} I_{0}^{t i p} \hat{W}_{r}(L) \frac{\mathrm{d} \hat{W}_{q}(L)}{\mathrm{d} x} \dot{w}(t) \\
& +c_{v} I_{2}^{t i p} \frac{\mathrm{d} \hat{W}_{r}(L)}{\mathrm{d} x} \frac{\mathrm{d} \hat{W}_{q}(L)}{\mathrm{d} x} \dot{w}(t)+\int_{0}^{L} c_{d} C_{s} \frac{\mathrm{d}^{2} \hat{W}_{r}(x)}{\mathrm{d} x^{2}} \frac{\mathrm{d}^{2} \hat{W}_{q}(x)}{\mathrm{d} x^{2}} \mathrm{~d} x \dot{w}_{r}(t) \\
& +\int_{0}^{L} C_{s} \frac{\mathrm{d}^{2} \hat{W}_{r}(x)}{\mathrm{d} x^{2}} \frac{\mathrm{d}^{2} \hat{W}_{q}(x)}{\mathrm{d} x^{2}} \mathrm{~d} x w_{r}(t)+\mu \frac{\mathrm{d} \hat{W}_{q}(L)}{\mathrm{d} x} v(t) \\
& =-\int_{0}^{L} I_{0} \hat{W}_{q}(x) \mathrm{d} x \ddot{w}_{\text {base }}(t)-I_{0}^{t i p} \hat{W}_{q}(L) \ddot{w}_{\text {base }}(t)-x_{c} I_{0}^{t i p} \frac{\mathrm{d} \hat{W}_{q}(L)}{\mathrm{d} x} \ddot{w}_{\text {base }}(t) .
\end{aligned}
$$


Since parameters $\hat{W}_{\mathrm{r}}(x)$ and $\hat{W}_{\mathrm{q}}(x)$ indicate normalised mode shapes, the orthonormality property from Eq. (67) can be proved by applying the orthogonality property of the mechanical dynamic equations to give,

$$
\begin{aligned}
& \int_{0}^{L} I_{0} \hat{W}_{r}(x) \hat{W}_{q}(x) \mathrm{d} x+I_{0}^{t i p} \hat{W}_{r}(L) \hat{W}_{q}(L)+x_{c} I_{0}^{t i p} \frac{\mathrm{d} \hat{W}_{r}(L)}{\mathrm{d} x} \hat{W}_{q}(L)+x_{c} I_{0}^{t i p} \hat{W}_{r}(L) \frac{\mathrm{d} \hat{W}_{q}(L)}{\mathrm{d} x} \\
& +I_{2}^{t i p} \frac{\mathrm{d} \hat{W}_{r}(L)}{\mathrm{d} x} \frac{\mathrm{d} \hat{W}_{q}(L)}{\mathrm{d} x}=\delta_{r q}=\left\{\begin{array}{lll}
0 & \text { if } & r \neq q \\
1 & \text { if } r=q
\end{array},\right. \\
& \int_{0}^{L} C_{s} \frac{\mathrm{d}^{2} \hat{W}_{r}(x)}{\mathrm{d} x^{2}} \frac{\mathrm{d}^{2} \hat{W}_{q}(x)}{\mathrm{d} x^{2}} \mathrm{~d} x=\omega_{r}{ }^{2} \delta_{r q}=\left\{\begin{array}{ccc}
0 & \text { if } & r \neq q \\
\omega_{r}{ }^{2} & \text { if } r=q
\end{array},\right.
\end{aligned}
$$

Two Rayleigh mechanical damping coefficients can separately be formulated from Eq. (67) by applying orthonormality. The mass proportional damping terms due to air friction can be formulated as,

$$
\begin{aligned}
& \int_{0}^{L} c_{v} I_{0} \hat{W}_{r}(x) \hat{W}_{q}(x) \mathrm{d} x+c_{v} I_{0}^{t i p} \hat{W}_{r}(L) \hat{W}_{q}(L)+x_{c} c_{v} I_{0}^{t i p} \frac{\mathrm{d} \hat{W}_{r}(L)}{\mathrm{d} x} \hat{W}_{q}(L) \\
& +x_{c} c_{v} I_{0}^{t i p} \hat{W}_{r}(L) \frac{\mathrm{d} \hat{W}_{q}(L)}{\mathrm{d} x}+c_{v} I_{2}^{t i p} \frac{\mathrm{d} \hat{W}_{r}(L)}{\mathrm{d} x} \frac{\mathrm{d} \hat{W}_{q}(L)}{\mathrm{d} x}=c_{v} \delta_{r q}= \begin{cases}0 & \text { if } r \neq q \\
c_{v} & \text { if } r=q\end{cases}
\end{aligned}
$$

The stiffness proportional damping terms due to internal friction of damping stress for the laminated piezoelectric structure from Eq. (67) can be formulated as,

$$
\int_{0}^{L} c_{d} C_{s} \frac{\mathrm{d}^{2} \hat{W}_{r}(x)}{\mathrm{d} x^{2}} \frac{\mathrm{d}^{2} \hat{W}_{q}(x)}{\mathrm{d} x^{2}} d x=c_{d} \omega_{r}{ }^{2} \delta_{r q}=\left\{\begin{array}{ll}
0 & \text { if } r \neq q \\
c_{d} \omega_{r}{ }^{2} & \text { if } r=q
\end{array} .\right.
$$

Therefore, Rayleigh mechanical damping coefficient can simply be reduced as,

$$
c_{r q}=c_{v} \delta_{r q}+c_{d} \omega_{r}^{2} \delta_{r q}=2 \zeta_{r} \omega_{r} \delta_{r q}
$$

where $c_{v}$ and $c_{d}$ indicate mass proportional damping coefficient and stiffness proportional damping coefficient, respectively. Corresponding to Eqs. (68)-(69), equation (67) can now be reformulated by including the Rayleigh mechanical damping from Eq. (72), the result of which can be coupled with Eq. (63) to give the normalised closed-form electromechanical transverse dynamic equations with input base excitation as,

$$
\begin{gathered}
\ddot{w}_{r}(t)+2 \zeta_{r} \omega_{r} \dot{w}_{r}(t)+\omega_{r}{ }^{2} w(t)+P_{r} v(t)=-Q_{r} \ddot{w}_{\text {base }}(t), \\
\sum_{r=1}^{\infty} \hat{P}_{r}^{(w)} \dot{w}_{r}(t)+P_{D} \dot{v}(t)+\frac{v(t)}{R_{\text {load }}}=0 .
\end{gathered}
$$


It is noted that because equation (73) has been normalised, the parameters $P_{r}, \hat{P}_{r}, P_{D}$, and $Q_{r}$ can be reduced as,

$$
\begin{gathered}
P_{r}=\mu \frac{\mathrm{d} \hat{W}_{r}}{\mathrm{~d} x}(L), \quad \sum_{r=1}^{\infty} \hat{P}_{r}=-\sum_{r=1}^{\infty} \int_{0}^{L} \mu \frac{\mathrm{d}^{2} \hat{W}_{r}(x)}{\mathrm{d} x^{2}} \mathrm{~d} x, \\
P_{D}=\int_{0}^{L} C_{p} \mathrm{~d} x, \quad Q_{r}=\int_{0}^{L} I_{0} \hat{W}_{r}(x) \mathrm{d} x+I_{0}^{t i p} \hat{W}_{r}(L)+x_{c} I_{0}^{t i p} \frac{\mathrm{d} \hat{W}_{r}(L)}{\mathrm{d} x} .
\end{gathered}
$$

The second multi-mode FRF is the transverse motion with respect to input motions. If baseinput transverse motion is ignored, the FRF of transverse motion related to the base input longitudinal motion can be obtained as,

$$
\frac{w_{r}(j \omega)}{-\omega^{2} w_{\text {base }} e^{j \omega t}}=-\frac{1}{\omega_{r}^{2}-\omega^{2}+j 2 \zeta_{r} \omega_{r} \omega}\left(\frac{\left(j \omega P_{D}+\frac{1}{R_{\text {load }}}\right) Q_{r}}{j \omega P_{D}+\frac{1}{R_{\text {load }}}-\sum_{r=1}^{\infty} \frac{j \omega \hat{P}_{r} P_{r}}{\omega_{r}^{2}-\omega^{2}+j 2 \zeta_{r} \omega_{r} \omega}}\right),
$$

The multi-mode FRF of transverse displacement with respect to input base transverse acceleration can be obtained as,

$$
\frac{w(x, j \omega)}{-\omega^{2} w_{\text {base }} e^{j \omega t}}=-\sum_{r=1}^{\infty}\left[\frac{\hat{W}_{r}(x)}{\omega_{r}{ }^{2}-\omega^{2}+j 2 \zeta_{r} \omega_{r} \omega}\left(\frac{\left(j \omega P_{D}+\frac{1}{R_{\text {load }}}\right) Q_{r}}{\left.j \omega P_{D}+\frac{1}{R_{\text {load }}}-\sum_{r=1}^{\infty} \frac{j \omega \hat{P}_{r} P_{r}}{\omega^{2}-\omega^{2}+j 2 \zeta_{r} \omega_{r} \omega}\right)}\right],\right.
$$

The absolute transverse displacement and velocity FRFs can be also be formulated as,

$$
\begin{gathered}
\frac{w_{a b s}(x, \mathrm{j} \omega)}{-\omega^{2} w_{b a s e} e^{\mathrm{j} \omega t}}=-\frac{1}{\omega^{2}}+\frac{w(x, \mathrm{j} \omega)}{-\omega^{2} w_{b a s e} e^{\mathrm{j} \omega t}}, \\
\frac{\dot{w}_{a b s}(x, \mathrm{j} \omega)}{-\omega^{2} w_{\text {base }} e^{\mathrm{j} \omega t}}=\frac{1}{\mathrm{j} \omega}+\mathrm{j} \omega \frac{w(x, \mathrm{j} \omega)}{-\omega^{2} w_{\text {base }} e^{\mathrm{j} \omega t}} .
\end{gathered}
$$

The multi-mode FRF of transverse displacement with respect to input base transverse acceleration can be obtained as,

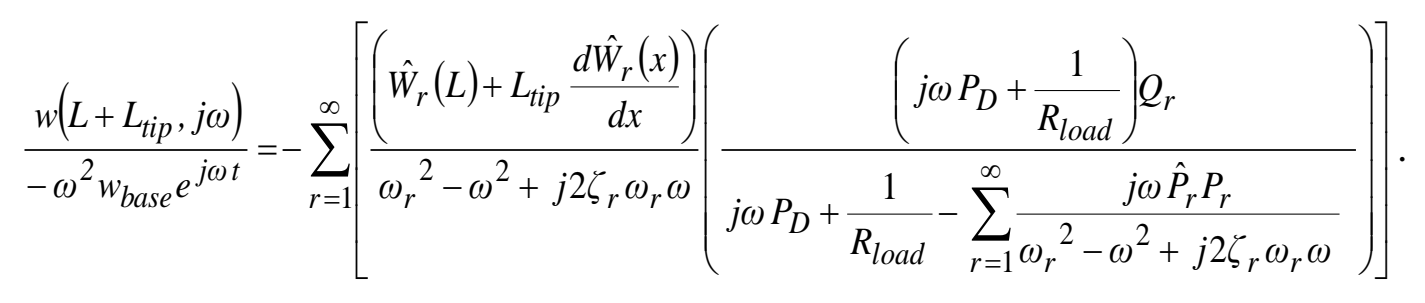

The absolute transverse displacement and velocity FRFs at any position along the proof mass offset can be formulated as, 


$$
\begin{gathered}
\frac{w_{a b s}\left(L+L_{t i p}, \mathrm{j} \omega\right)}{-\omega^{2} w_{\text {base }} e^{\mathrm{j} \omega t}}=-\frac{1}{\omega^{2}}+\frac{w\left(L+L_{t i p}, \mathrm{j} \omega\right)}{-\omega^{2} w_{b a s e} e^{\mathrm{j} \omega t}}, \\
\frac{\dot{w}_{a b s}\left(L+L_{t i p}, \mathrm{j} \omega\right)}{-\omega^{2} w_{\text {base }} e^{\mathrm{j} \omega t}}=\frac{1}{\mathrm{j} \omega}+\mathrm{j} \omega \frac{w\left(L+L_{t i p}, \mathrm{j} \omega\right)}{-\omega^{2} w_{\text {base }} e^{\mathrm{j} \omega t}} .
\end{gathered}
$$

The multi-mode FRF between electric voltage output and the input base transverse acceleration can be obtained as,

$$
\frac{v_{p}(j \omega)}{-\omega^{2} w_{\text {base }} e^{j \omega t}}=\frac{\sum_{r=1}^{\infty} \frac{j \omega \hat{P}_{r} Q_{r}}{\omega_{r}^{2}-\omega^{2}+j 2 \zeta_{r} \omega_{r} \omega}}{j \omega P_{D}+\frac{1}{R_{\text {load }}}-\sum_{r=1}^{\infty} \frac{j \omega \hat{P}_{r} P_{r}}{\omega_{r}{ }^{2}-\omega^{2}+j 2 \zeta_{r} \omega_{r} \omega}} .
$$

The multi-mode FRF of the electric current output related to the input base transverse acceleration can be derived, where the base input longitudinal acceleration is omitted to give,

$$
\frac{i_{p}(j \omega)}{-\omega^{2} w_{\text {base }} e^{j \omega t}}=\frac{\frac{1}{R_{\text {load }}} \sum_{r=1}^{\infty} \frac{j \omega \hat{P}_{r} Q_{r}}{j \omega P_{D}{ }^{2}-\frac{1}{R_{\text {load }}}-\omega^{2}+j 2 \zeta_{r} \omega_{r} \omega}-\sum_{r=1}^{\infty} \frac{j \omega \hat{P}_{r} P_{r}}{\omega_{r}^{2}-\omega^{2}+j 2 \zeta_{r} \omega_{r} \omega}}{.}
$$

The multi-mode FRF of power harvesting related to the input transverse acceleration can be derived as,

$$
\frac{P_{p}(j \omega)}{\left(-\omega^{2} w_{\text {base }} e^{j \omega t}\right)^{2}}=\left[\frac{\frac{1}{\sqrt{R_{\text {load }}}} \sum_{r=1}^{\infty} \frac{j \omega \hat{P}_{r} Q_{r}}{\omega_{r}{ }^{2}-\omega^{2}+j 2 \zeta_{r} \omega_{r} \omega}}{j \omega P_{D}+\frac{1}{R_{\text {load }}}-\sum_{r=1}^{\infty} \frac{j \omega \hat{P}_{r} P_{r}}{\omega_{r}{ }^{2}-\omega^{2}+j 2 \zeta_{r} \omega_{r} \omega}}\right]^{2} .
$$

To obtain the optimal multi-mode FRF power harvesting, equation (82) can be differentiated with respect to load resistance and the differentiable power function can be set to zero to give the optimal load resistance. Corresponding to Eq. (82) the optimal load resistance can be formulated as,

$$
R_{\text {load }}^{\text {opt }}=\frac{\sqrt{X(\omega)^{2}+Y(\omega)^{2}}}{X(\omega)^{2}+Y(\omega)^{2}}
$$

where

$$
X(\omega)=\omega P_{D}-\sum_{r=1}^{\infty} \frac{\omega P_{r}{ }^{2}\left(\omega_{r}{ }^{2}-\omega^{2}\right)}{\left(\omega_{r}{ }^{2}-\omega^{2}\right)^{2}+\left(2 \zeta_{r} \omega_{r} \omega\right)^{2}}
$$




$$
Y(\omega)=\sum_{r=1}^{\infty} \frac{\omega P_{r}^{2}\left(2 \zeta_{r} \omega_{r} \omega\right)}{\left(\omega_{r}^{2}-\omega^{2}\right)^{2}+\left(2 \zeta_{r} \omega_{r} \omega\right)^{2}} .
$$

It should be noted that the optimal load resistance can be substituted back into Eq. (82) to give the optimal power harvesting.

\section{Result and Discussion}

Comprehensive case studies of the numerical and analytical validations including the experimental results are discussed using the electromechanical FRFs with variable load resistance. This section discusses three important parts. For the first part, the computational capability of the novel numerical techniques in comparison with analytical models is discussed in terms of the dynamic system responses. This also includes the experimental validation. For the second part, the proposed numerical techniques enable the prediction of optimal power harvesting response and frequency bandwidth for identifying the performance obtainable by varying the piezoelectric thickness and physical property optimisations with distributed piezoelectric element. For the third part, the proposed numerical techniques enable the prediction of optimal power harvesting response for identifying the parametric design optimisations with segmented piezoelectric elements.

\subsection{Numerical, analytical and experimental validations of the modal damped vibrations of the distributed piezoelectric unimorph with the proof mass offset.}

This section discusses validation of three different studies using the numerical, analytical and experimental results for the modal damped vibration response of the distributed piezoelectric unimorph beam with the proof mass offset. The selected piezoelectric properties made from PZT PSI-5A4E (Piezo Systems, Inc) are listed in Table 1. The input base transverse acceleration onto the cantilevered piezoelectric unimorph beam was chosen to be 1 $\mathrm{m} / \mathrm{s}^{2}$. In Fig. 3, the device length $L$ and width $b$ with piezoelectric thickness $h_{p}$ and substructure (brass) thickness $h_{s}$ were set to $60 \mathrm{~mm}, 6 \mathrm{~mm}, 0.127 \mathrm{~mm}$ and $0.5 \mathrm{~mm}$, respectively. The proof mass configurations of the piezoelectric structure were calculated according to the geometry and material property made from steel where the dimensions of proof mass with length $l_{t}$, thickness $h_{t}$ and width $w_{t}$ (width) were set to $15 \mathrm{~mm}, 10 \mathrm{~mm}$ and 6 $\mathrm{mm}$, respectively. Offset distances of proof mass $\bar{x}_{c}$ and $\bar{z}_{c}$ can be found in Appendix A. Note that the extra length of the substructure glued on the proof mass was assumed to be a body mass contributing the proof mass offset. Moreover, the complete experimental setup as 
shown in Fig. 4 was utilised for further validations. The results shown in Fig. 5a include the absolute tip velocity FRFs at the first mode using the three different methods show very good agreement under the variable load resistance. As can be seen, the higher amplitudes can be achieved at the short and open circuit resonance frequencies of $18.5 \mathrm{~Hz}$ and $18.9 \mathrm{~Hz}$ when the load resistances approach the lower and higher values (from short to open circuit load resistances), respectively.

Further validations of the electromechanical FRFs can be seen in Figs. 5b-5d. Since our main concern is to present the validations of the novel mathematical studies (numerical and analytical) with special emphasis on the modal damping of the normalised dynamic systems of the piezoelectric unimorph beam and tip offset, the trends of electromechanical FRFs can be found to very similar with the established facts of the previous analytical literatures [7][9]. In electromechanical FRFs, the damping effects of the system consist of mechanical damping, electromechanical damping and electrical damping [8]. Mechanical damping ratio at first mode $\zeta_{1}=0.0162$ was identified by fitting the results obtained from the measurement and theoretical methods using the velocity FRF with the load resistance approaching to short circuit in order to obtain accurate results. On the other hand, the electromechanical damping effect can be found in the piezoelectric coupling and piezoelectric capacitance that can be seen in Eqs. (29) and (33) whereas the electrical damping can be found in the resistive shunt circuit. Again, the voltage, current and power FRFs with the variable load resistance given from the numerical and analytical methods gave very accurate results compared to the experimental results. The shifting frequencies from short to open circuit load resistances can also tune the amplitude levels for each FRFs. Moreover, Fig. 6a shows that the maximum power amplitude can be captured at certain levels of the increasing velocity amplitude with different frequency responses when the load resistance moves from short to open circuits. In other words, the maximum power harvesting does not mean the system response has maximum velocity. More noticeably, maximum power can be achieved at frequencies between $18.43 \mathrm{~Hz}$ and $19.05 \mathrm{~Hz}$. For better indication, the highest power output as shown in Fig. $5 \mathrm{~d}$ can be seen at the short and open circuit resonance frequencies at precise values of 18.5 Hz and $18.9 \mathrm{~Hz}$, respectively. By viewing a particular location as shown in Fig. 6b, the power amplitudes at off-resonances, with the load resistance moving from short to open circuits, increase gradually until reaching the highest level and then decrease to the lowest level, followed by increasing velocity amplitudes. However, when the system response approaches the short and open circuit resonance frequencies, the power amplitudes with the 
load resistance moving from short to open circuits, increase rapidly with a slight decrease of velocity amplitude before reaching the highest level of power, and then decrease slowly to the minimum point, followed by increasing velocity amplitude. Moreover, Fig. $6 \mathrm{~b}$ also shows that the maximum power amplitude trends with different frequency responses can be seen at certain levels of decreasing current amplitude when the load resistance moves from short to open circuits. At this particular situation, the highest power tends to approach the short and open circuit resonance frequencies with a gradual decrease of velocity amplitudes, followed by increasing load resistances from short to open circuits.

As mentioned previously, the proposed novel numerical technique introduced the 1-D laminated beam element where most of the multi-physics finite element softwares only provide the 2-D and 3-D coupled-field elemental attribute facilities for meshing piezoelectric beam structures [32]- [33]. The proposed electromechanical finite element vibration shows considerable convenience, once the matrix equations of the electromechanical discretised element were developed and analysed using a MATLAB program. The technical challenge depends on the computational efficiency in developing the auto-generation computing program codes for the multi-element formulation. Once the program codes were developed and tested for correctness, the proposed numerical technique can be used for analysing the parametric case studies with different geometrical aspects and physical properties as further discussed in the next section. As a result, it shows reliable and convenient computational process. In Table 2, it can be seen that the CPU time of power harvesting FRFs was slightly higher than the natural frequency because the FRFs using Eq. (36) requires an iterative process that depends on the frequency step size, number of degrees of freedom and piezoelectric elements. Note that the computer system for running the simulation was an Intel core i7-4770 CPU $3.40 \mathrm{GHz}$ with $16 \mathrm{~GB}$ RAM. In this paper, the power FRFS with 9 different load resistance values have used frequency step of $0.1 \mathrm{~Hz}$ spanning from $10 \mathrm{~Hz}-30$ Hz. Moreover, iterating the natural frequency using the expression, $\left(\boldsymbol{K}-\omega^{2} \boldsymbol{M}\right) \boldsymbol{U}$ is quite straightforward because Matlab has common commands for analysing eigenvectors (d) for mode shapes and eigenvalues for natural frequencies (v) from the global matrix A using $[\mathrm{d}, \mathrm{v}]=\mathrm{eig}(\mathrm{A})$. Overall, the computational cost during the process of each iteration for 50 elements only takes less than 10 seconds for the power FRFs and 3 seconds for the natural frequencies. 
On the other hand, the analytical technique as the exact analytical method depends on the solutions of the partial differential equations with the proper boundary conditions where dynamic response of the electromechanical piezoelectric structures depends on identifying the frequency equations and eigenfunction solutions. Once these are identified, the electromechanical FRFs can be formulated and analysed. However, the computational process will be challenging, if the geometrical parameters (length, thickness, properties, etc) are varied. Moreover, if the complex structures such as the segmented piezoelectric structures onto the substructure are applied, the computational process will be even more tedious and challenging.

\subsection{Parametric design and physical properties of the modal damped vibrations of the distributed piezoelectric unimorph with the proof mass offset.}

Discussion on the optimal power harvesting FRFs using different physical piezoelectric properties are presented using the numerical technique for identifying the optimal frequency bandwidths and for analysing the vibration characteristics of the parametric design optimisation. Note that mechanical damping ratio $\zeta_{1}=0.0162$ as shown in section 4.1 was used on this case where it was obtained using the chosen Rayleigh damping coefficients of $2.856 \mathrm{rad} / \mathrm{s}\left(c_{v}\right)$ and $6.727 \mathrm{e}-5 \mathrm{~s} / \mathrm{rad}\left(c_{d}\right)$. The investigation of the optimal power harvesting FRFs using different material properties from Table 3 can be seen in Fig. 7a, where each material shows different operating frequency bandwidths and resonance frequencies. This can be seen clearly in Fig. 7b, the frequency bandwidths for each optimal power output show the different size due to strong effect of different piezoelectric electromechanical coupling. As can be seen, the PZN-PT material shows very high piezoelectric constant resulting in the strongest electromechanical coupling where the operating frequency bandwidth give the highest value among other piezoelectric materials because there are two peaks of equal amplitude from the PZN-PT optimal power response resulting in the wider frequency band. On the other hand, PVDF shows the weakest electromechanical coupling due to very low piezoelectric constant. Note that the example in [34]-[35] also shows the similar application of discussing strong and weak electromechanical coupling using different piezoelectric constants where the studies also show relevancy of this section using our novel theoretical studies. At this point, the frequency bandwidths for each piezoelectric power harvesting device show benefit for identifying the performance of the electromechanical system. Moreover, parametric design of piezoelectric thickness with the chosen piezoelectric 
materials can also be used to widen the frequency bandwidth as shown in Figs. 8a-d. Only piezoelectric thickness was varied where other physical properties and geometries remain constant. It is clearly seen that increasing piezoelectric thickness may also contribute to increase in the frequency bandwidth. The optimal power harvesting FRF as shown in Fig. 8 was calculated using Eqs. (36) and (37). It can be seen that the transition between weak and strong electromechanical couplings occurs when the piezoelectric thickness increases slightly. For example, for the PZN-PT material with the particular piezoelectric thicknesses, the two amplitude peaks of the optimal power FRF was obtained using the optimal load resistances. It means that the power FRF amplitudes with certain load resistance coincident with the two peaks of the optimal power FRF have different resonances for each single peak. For this point, the lower resonance frequency for the first amplitude peak is obviously the same as the natural frequency of the mechanical system. Moreover, the higher resonance frequency for the second peak is actually a shifting frequency due to the effect of the electromechanical system consisting of piezoelectric coupling and capacitance and resistive shunt circuit. In essence, the natural frequency and eigenvectors only depend on the characteristics of the mechanical system since they are obtained from the expression, $\left(\boldsymbol{K}-\omega^{2} \boldsymbol{M}\right) \boldsymbol{U}$. Moreover, for coupled system behaviour from the power harvesting system, the nature of the mechanical system of the piezoelectric structure can be affected by the nature of the electromechanical system of the piezoelectric itself including the addition of the load resistance. Therefore, the behaviour of the two amplitude peaks of the piezoelectric structure has strong electromechanical coupling. The lower and higher resonance frequencies for the two peaks are sometime called the short and open circuit resonances, respectively. Note that the short circuit resonance is the same as the natural frequency of the system [2, 8, 10, 27]. If the thickness of piezoelectric is reduced until giving single peak of amplitude, the resonance frequency of the optimal power FRF is the same as the natural frequency of the mechanical system having the equivalent eigenvectors. At this point, the piezoelectric structure has weak electromechanical coupling. Nevertheless, the actual eigenvectors including eigenvalues reduced from mechanical system can be used as reference for investigating the behaviour of electromechanical frequency response.

Further detail of frequency bandwidth differences for each piezoelectric material can be seen in Fig. 9a. As a function of thickness, the maximum power amplitude as shown in Fig. $9 \mathrm{~b}$ can also be obtained for each material. Only PVDF material shows the lowest amplitude value although the thickness increases. Note that if the input vibration applied onto the 
piezoelectric unimorph beam is kept constant, further increasing piezoelectric thickness might result in a decrease of the optimal power amplitude significantly and also might invalidate the thin beam Euler-Bernoulli condition (ratio between beam length to thickness at the minimum order of 20). Further studies of the effect of the piezoelectric thickness including other parametric designs in the system response of the optimal power harvesting can be discussed in the next stage.

\subsection{Parametric design of PSI-5A4E of the modal damped vibrations of the segmented piezoelectric unimorph with the proof mass offset.}

Parametric design-based electromechanical optimal power harvesting using the variations of piezoelectric length $\left(x_{d i v}\right)$, thickness $\left(h_{p}\right)$ and capacitance $\left(P_{D}\right)$ and proof mass length $\left(l_{\text {tip }}\right)$ can be further explored in order to identify the particular locations of the maximum power using the numerical technique. It is noted that the geometry of the substrate as given earlier remains constant where the segmented piezoelectric coverage was measured from the base to the end of the beam as shown in Fig. 10. For this case, piezoelectric material PSI-5A4E was chosen because the material was also used in the experimental studies as given section 4.1. Note that the identification of maximum power using parametric geometrical design was based on the given formula of numerical studies as shown in Eqs. (36)-(37) where it shows the optimal power harvesting FRF based on the optimal load resistance. In Figs. 11a-d, the region of producing maximum power harvesting using the parametric design can be seen by increasing piezoelectric thicknesses and lengths of the portion of the piezoelectric segment lengths between $48 \mathrm{~mm}$ and $60 \mathrm{~mm}$ with the thicknesses between $0.127 \mathrm{~mm}$ and $0.197 \mathrm{~mm}$. In that region, the maximum power harvesting with the frequency ranges from $15 \mathrm{~Hz}$ to 20 $\mathrm{Hz}$ and damping ratios from 0.016 to 0.018 can be identified with the higher internal capacitance reaching up to $90 \mathrm{nF}$. Note that varying mechanical damping ratio based on the parametric geometrical design was calculated using the chosen Rayleigh damping coefficients of $2.856 \mathrm{rad} / \mathrm{s}\left(c_{v}\right)$ and $6.727 \mathrm{e}-5 \mathrm{~s} / \mathrm{rad}\left(c_{d}\right)$. This shows that the input base transverse motion onto the piezoelectric beam structure can create the bending motion of the elemental beam resulting in the induction of the electric and polarity fields of the piezoelectric element to be even more sensitive. At this case, the piezoelectric coupling with 3-1 mode of operation is the most suitable response for generating the maximum power output under bending mode. However, low power output can be obtained, if the piezoelectric thickness and length increase continuously because that will result in larger dynamical ratio 
between stiffness and mass of the piezoelectric structure producing higher resonance frequency with very low damping ratio.

Further parametric studies using the variances of the proof mass length and piezoelectric length can also be seen in Figs. 12a-c. The power outputs of the system responses show maximum level with lower resonance frequencies and larger mechanical damping ratios when increasing the volume of the proof mass and the piezoelectric segment lengths at the certain dimension. The largest mechanical damping ratio can be obtained when the volume of proof mass increases with reduction of piezoelectric length resulting in the lowest resonance frequency with a relative higher power amplitude. Note that since the increasing proof mass geometry contributes to the mass matrix of the numerical solution, it directly affects the mass proportional Rayleigh damping coefficient giving the larger mechanical damping ratio. It is obvious to see that the resonance frequency can be larger value, if the volume of the proof mass reduces slightly. However, in this case, the optimal power amplitude does not give the maximum value. It can be arguably stated that most of the typical power harvesting devices have attached the proof mass in order to give higher power amplitude, especially to tune the lower frequency response that fits to the vibration environment. Overall, the investigation of the parametric design of the power harvester device with variable proof mass and piezoelectric geometries can be used to identify the maximum power output with low resonance frequency.

\section{Conclusion}

Expressions of mathematical techniques using electromechanical finite element analysis and analytical closed-form boundary value method have been presented in this paper with particular emphasis on the modal damped vibration system responses of the piezoelectric power harvesting with dynamical proof mass offset. Matrix electromechanical finite element dynamic equations reduced from the extended Lagrangian principle were further formulated using orthonormalised scalar forms to give EFRFs of voltage, current, power and velocity. On the other hand, analytical equations reduced from the variational principle based on the integro-differential equations were also further developed using the orthonormalised closedform boundary value methods to give EFRFs of voltage, current, power and velocity. Note that EFRFs reduced from numerical and analytical techniques show distinct equations that facilitate computational processes. The numerical techniques provide the benefits for 
analysing the electromechanical energy harvesters with different geometry and scalability of devices that can reduce the complexity of solving the analytical techniques based on the integro-differential equations associated with their boundary conditions. The only challenge of the numerical techniques is the process of developing computational program codes, for example using the Matlab software. Once these codes show capability and accuracy of displaying the results from their post-processing systems, the numerical techniques can provide effective and quick predictions for analysing various case studies. On the other hand, the analytical techniques proposed here provide complementary methods for the use of validation as required by numerical techniques.

The result shows that the system responses from numerical and analytical studies give excellent agreement to that of experimental result. Further parametric geometrical design and physical properties of the piezoelectric power harvesters have been presented using numerical EFRFs. The result shows that the analysis of the optimal frequency bandwidth can be a useful technique for investigating weak and strong electromechanical effects and optimal responses of the various piezoelectric properties including different geometrical designs of piezoelectric structure and proof mass. These parametric studies provide the benefit for identifying the maximum power output, low resonance frequency and larger frequency bandwidth because the studies can be used to identify the performance of the device based on the best-fit amplitude from the vibration environment.

\section{Appendix A. Mass moment of inertias of the unimorph beam and proof mass offset}

Coefficient mass moments of inertia can simply be formulated based on geometry and material property of the piezoelectric bimorph. The zeroth mass moment of inertia of the unimorph beam was given as,

$$
I_{0}=\rho^{(1)} b^{(1)} h^{(1)}+\rho^{(2)} b^{(2)} h^{(2)} .
$$

The mass moment of inertias of the proof mass offset as shown in Fig. 3 can be formulated. Note that the extra unimorph beam length also contributed to the proof mass offset. The zeroth mass moment of inertia can be stated as,

$$
I_{0}^{t i p}=\rho^{t i p} b l_{t} h_{t}+\rho^{(1)} b l_{t} h_{s},
$$

and the second mass moment of inertia of proof mass offset at the end of unimorph beam with the coincided point of neutral axis $d$ as shown in Fig. 3 can be formulated as, 


$$
\begin{aligned}
I_{2}^{t i p} & =\rho^{t i p} b l_{t} h_{t}\left(\frac{\left(l_{t}{ }^{2}+h_{t}^{2}\right)}{12}+\left(\frac{h_{t}}{2}-h_{p}-\bar{z}_{c}+z_{n}\right)^{2}\right) \\
& +\rho^{(1)} b l_{t} h_{s}\left(\frac{\left(l_{t}^{2}+h_{s}^{2}\right)}{12}+\left(\frac{h_{s}}{2}+h_{p}-z_{n}+\bar{z}_{c}\right)^{2}\right)+I_{0}^{t i p}\left(\bar{z}_{c}{ }^{2}+\bar{x}_{c}{ }^{2}\right),
\end{aligned}
$$

where the offset distances measured from the proof mass centroid to the point $d$ in the $\mathrm{x}$-and $z$-axes can respectively be formulated as,

$$
\begin{gathered}
\bar{x}_{c}=\frac{\rho^{t i p} b l_{t}{ }^{2} h_{t}+\rho^{(1)} b l_{t}{ }^{2} h_{s}}{2\left(\rho^{t i p} b l_{t} h_{t}+\rho^{(1)} b l_{t} h_{s}\right)}, \\
\bar{z}_{c}=\frac{\rho^{t i p} b l_{t} h_{t}\left(\frac{h_{t}}{2}+z_{n}-h_{p}\right)+\rho^{(1)} b l_{t}\left(z_{n}-h_{p}\right)\left(\frac{z_{n}-h_{p}}{2}\right)-\rho^{(1)} b l_{t}\left(h_{s}+h_{p}-z_{n}\right)\left(\frac{h_{s}+h_{p}-z_{n}}{2}\right)}{\rho^{t i p} b l_{t} h_{t}+\rho^{(1)} b l_{t}\left(z_{n}-h_{p}\right)+\rho^{(1)} b l_{t}\left(h_{s}+h_{p}-z_{n}\right)} .
\end{gathered}
$$

\section{Appendix B. Stiffness coefficients for the unimorph beam}

The total transverse stiffness coefficient for two layers can be formulated as,

$$
C_{s}=\frac{b}{3}\left(\bar{c}_{11}^{(1)}\left(h_{p}+h_{s}-z_{n}\right)^{3}-\bar{c}_{11}^{(1)}\left(h_{p}-z_{n}\right)^{3}+\bar{c}_{11}^{(2)}\left(h_{p}-z_{n}\right)^{3}+\bar{c}_{11}^{(2)} z_{n}^{3}\right)
$$

\section{Appendix C. Transverse piezoelectric coupling coefficient and internal capacitance of piezoelectric}

It is noted that piezoelectric coupling $\mu$ comes from the converse and direct effect of the piezoelectric material respectively [27]. Transverse piezoelectric coupling can be formulated as,

$$
\mu=-\frac{e_{31} b\left(2 z_{n} h_{p}-h_{p}{ }^{2}\right)}{2 h_{p}} .
$$

The piezoelectric capacitance at the piezoelectric layer can be calculated as,

$$
C_{p}=\frac{\varepsilon_{33}^{S} b}{h_{p}} .
$$

\section{Appendix D. Mode shapes of the cantilevered unimorph beam with proof mass offset}

The normalised eigenfunction series $\hat{\Psi}_{r}(x)$ in Eq. (61), can be proved by manipulating Eqs. (58) and (60) and taking only consideration of the transverse mechanical equation of the typical Euler-Bernoulli unimorph beam with cantilevered model by substituting 
$w_{r e l}(x, t)=\sum_{r=1}^{\infty} \Psi_{r}(x) w_{r}(t)$. The reduced characteristic mechanical equation can be formulated to give,

$$
\left[\begin{array}{ll}
A_{11} & A_{12} \\
A_{21} & A_{22}
\end{array}\right]\left\{\begin{array}{l}
a_{1} \\
a_{4}
\end{array}\right\}=0
$$

where: $\quad A_{11}=-(\cos (\alpha L)+\cosh (\alpha L))+\frac{I_{2}^{t i p} \alpha^{3}}{I_{0}}(\sin (\alpha L)+\sinh (\alpha L))$

$$
\begin{aligned}
& -\frac{x_{c} I_{0}^{t i p} \alpha^{2}}{I_{0}}(\cos (\alpha L)-\cosh (\alpha L)) \\
A_{12}= & (\sin (\alpha L)+\sinh (\alpha L))+\frac{I_{2}^{t i p} \alpha^{3}}{I_{0}}(\cos (\alpha L)-\cosh (\alpha L)) \\
& +\frac{x_{c} I_{0}^{t i p} \alpha^{2}}{I_{0}}(\sin (\alpha L)-\sinh (\alpha L)), \\
A_{21}= & (\sin (\alpha L)-\sinh (\alpha L))+\frac{I_{0}^{t i p} \alpha}{I_{0}}(\cos (\alpha L)-\cosh (\alpha L)) \\
- & \frac{x_{c} I_{0}^{t i p} \alpha^{2}}{I_{0}}(\sin (\alpha L)+\sinh (\alpha L)), \\
A_{22}= & (\cos (\alpha L)+\cosh (\alpha L))-\frac{I_{0}^{t i p} \alpha}{I_{0}}(\sin (\alpha L)-\sinh (\alpha L)) \\
& -\frac{x_{c} I_{0}^{t i p} \alpha^{2}}{I_{0}}(\cos (\alpha L)-\cosh (\alpha L)) .
\end{aligned}
$$

The frequency equation and eigenvalues can be formulated from Eq. (D1) leading to nontrivial solutions as,

$$
A_{11} A_{22}-A_{21} A_{12}=0 \text {. }
$$

The mode shape or space-dependent eigenfunction of transverse bending can be formulated can be formulated as,

$$
W_{r}(x)=a_{1 r}\left(\cos (\alpha x)-\cosh (\alpha x)+\frac{A_{21}}{A_{22}}(\sin (\alpha x)-\sinh (\alpha x))\right) .
$$

Since equation (D3) contains variable $a_{1 r}$ as the transverse amplitude constant, the normalised mode shape can be formulated as,

$$
\hat{W}_{r}(x)=\frac{W_{r}(x)}{\left(\int_{0}^{L} I_{0} W_{r}(x)^{2} d x+I_{0}^{t i p} W_{r}(L)^{2}+2 x_{c} I_{0}^{t i p} W_{r}(L) \frac{\mathrm{d} W_{r}}{\mathrm{~d} x}(L)+I_{2}^{t i p}\left(\frac{\mathrm{d} W_{r}}{\mathrm{~d} x}(L)\right)^{2}\right)^{1 / 2}}, r=1,2, \ldots ., m,
$$




\section{References}

[1] N.G. Stephen, On energy harvesting from ambient vibration, J. Sound Vib. 293 (2006) 409-425.

[2] A. Erturk, D.J. Inman, Piezoelectric energy harvesting (Wiley, 2011)

[3] S. Roundy, P.K. Wright, A piezoelectric vibration based generator for wireless electronics, Smart Mater. Struct. 18 (2004) 1131-1142.

[4] J. Liang, W.-H Liao, Impedance modeling and analysis for piezoelectric energy harvesting Systems, IEEE/ASME Trans. Mechatronics 17 (2012) 1145-1157.

[5] Y. Liao, H. Sodano, Model of a single mode energy harvester and properties for optimal power generation, Smart Mater. Struct.17 (2008) 065026.

[6] M. Kim, M. Hoegen, J. Dugundji, B.L. Wardle Modeling and experimental verification of proof mass effects on vibration energy harvester performance, Smart Mater. Struct. 19 (2010) 045023.

[7] H. Wang, Q. Meng, Analytical modeling and experimental verification of vibrationbased piezoelectric bimorph beam with a tip-mass for power harvesting, Mech. Syst. Signal Proc. 36 (2013) 193-209.

[8] M.F. Lumentut, I.M. Howard, Analytical and experimental comparisons of electromechanical vibration response of a piezoelectric bimorph beam for power harvesting, Mech. Syst. Signal Proc. 36 (2013) 66-86.

[9] A. Erturk A, Assumed-modes modeling of piezoelectric energy harvesters: EulerBernoulli, Rayleigh, and Timoshenko models with axial deformations, Comp. Struct. 106-107 (2012) 214-227.

[10] A.M. Wickenheiser, Eigensolution of piezoelectric energy harvesters with geometric discontinuities: Analytical modelling and validation, J. Intel. Mat. Syst. Struct. 24 (2013) 729-744.

[11] M.F. Lumentut, I.M. Howard, Electromechanical piezoelectric power harvester frequency response modelling using closed-form boundary value methods, IEEE/ASME Trans. Mechatronics. 19 (2014) 32-44.

[12] M.F. Lumentut, L.A. Francis, I.M. Howard, Analytical techniques for broadband multielectromechanical piezoelectric bimorph beams with multifrequency power harvesting, IEEE Trans. Ultrason. Ferroelectr. Freq. Control 59 (2012) 1555-1568.

[13] H. Zhang, K. Afzalul, Design and analysis of a connected broadband multipiezoelectric-bimorph-beam energy harvester, IEEE Trans. Ultrason. Ferroelectr. Freq. Control 61 (2014) 1016-1023.

[14] F. Goldschmidtboeing and P. Woias, Characterization of different beam shapes for piezoelectric energy harvesting, J. Micromech. Microeng. 18 (2008 ) 104013. 
[15] L.M. Miller, E. Halvorsen, T. Dong, P.K. Wright, Modeling and experimental verification of low-frequency MEMS energy harvesting from ambient vibrations, J. Micromech. Microeng. 21 (2011) 045029.

[16] R. Andosca, T.G. McDonald, V. Genova, S. Rosenberg, J. Keating, C. Benedixen, J. $\mathrm{Wu}$, Experimental and theoretical studies on MEMS piezoelectric vibrational energy harvesters with mass loading, Sens. Actuators A 178 (2012) 76-87.

[17] A. Abdelkefi, M. R. Hajj, and A. H. Nayfeh, Piezoelectric energy harvesting from transverse galloping of bluff bodies, Smart Mater. Struct., 22 (2013) 015014.

[18] Y. Yang, L. Zhao, L. Tang, Comparative study of tip cross-sections for efficient galloping energy harvesting, Appl. Phys. Lett. 102 (2013) 064105.

[19] M. Naillon, R.H. Coursant, F. Besner, Analysis of piezoelectric structures by a finite element method, ACTA Electronica 25 341-362.

[20] S.Y. Wang 2004 A finite element model for the static and dynamic analysis of a piezoelectric bimorph, Int. J. Solids Struct. 41 (1983) 4075-4096.

[21] A. Benjeddou, Advances in piezoelectric finite element modelling of adaptive structural elements: a survey, Comp.Struct., 2000, 76, pp. 347-363.

[22] H.S. Tzou, C.I. Tzeng, Distributed piezoelectric sensor/actuator design for dynamic measurement/control of distributed parameter system: A piezoelectric finite element approach, J. Sound Vib. 138 (1983) 17-34.

[23] J.M. Moita, I.F.P. Correia, C.M.M. Soares, Active control of adaptive laminated structures with bounded piezoelectric sensors and actuators, Comp. Struct. 82 (2004) 1349-1358.

[24] O. Thomas, J.-F. Deü, J. Ducarne, Vibrations of an elastic structure with shunted piezoelectric patches: efficient finite element formulation and electromechanical coupling coefficients, Int. J. Numer. Methods Engng. 8 (2009) 235-268.

[25] N.G. Elvin, A.A. Elvin, A coupled finite element-circuit simulation model for analyzing piezoelectric energy generators, J. Intel. Mater. Syst. Struct. 20 (2009) 587-595.

[26] Y. Yang, L. Tang, Equivalent circuit modeling of piezoelectric energy harvesters, J. Intell. Mater. Syst. Struct. 20 (2009) 2223-2235.

[27] M.F. Lumentut, I.M. Howard, Electromechanical finite element modelling for dynamic analysis of a cantilevered piezoelectric energy harvester with tip mass offset under base excitations, Smart Mater. Struct. 23 (2014) 095037.

[28] Standards Committee of the IEEE Ultrasonics, Ferroelectrics, and Frequency Control Society, 1987, IEEE standard on piezoelectricity, IEEE/ANSI Std. 176-1987, New York. 
[29] A.M. Wickenheiser, Design optimization of linear and non-linear cantilevered energy harvesters for broadband vibrations, J. Intel. Mat. Syst. Struct. 22 (2011) 1213-1225.

[30] H.T. Banks, D.J. Inman, On Damping Mechanisms in Beams, ASME J. App.Mech. 58(1991) 716-723.

[31] R.W. Clough, J. Penzien, Dynamics of Structures (Wiley 1975), New York.

[32] Coupled-field analysis guide, http://orange.engr.ucdavis.edu/Documentation12.1/121/ ans_cou.pdf\#page=40\&zoom=auto,32.4,569.295

[33] Piezoelectricity in ansys mechanical, say goodbye to command snippets!, http://www.ansys-blog.com/tag/piezoelectricity/

[34] M. Zhu, E. Worthington, J. Njuguna, Analyses of power output of piezoelectric energyharvesting devices directly connected to a load resistor using a coupled piezoelectriccircuit finite element method, IEEE Trans. Ultrason. Ferroelectr. Freq. Control 56 (2009) 1309-1318.

[35] Y. Liao, H. Sodano, Structural effects and energy conversion efficiency of power harvesting, J. Intel. Mat. Syst. Struct. 20 (2009) 505-514. 
Table 1. Properties of the piezoelectric unimorph system.

\begin{tabular}{lll}
\hline Material properties & Piezoelectric & Brass \\
\hline Young's modulus, $\bar{c}_{11}(\mathrm{GPa})$ & 66 & 105 \\
Density, $\rho\left(\mathrm{kg} / \mathrm{m}^{3}\right)$ & 7800 & 9000 \\
Piezoelectric constant, $d_{31}(\mathrm{pm} / \mathrm{V})$ & -190 & - \\
Permittivity, $\varepsilon_{33}^{T}(\mathrm{~F} / \mathrm{m})$ & $1800 \varepsilon_{\mathrm{o}}$ & - \\
permittivity of free space, $\varepsilon_{\mathrm{o}}(\mathrm{pF} / \mathrm{m})$ & 8.854 & - \\
\hline
\end{tabular}

Table 2. Computational cost based on the number of meshed elements.

\begin{tabular}{ccc}
$\begin{array}{l}\text { Number of } \\
\text { Elements }\end{array}$ & $\begin{array}{l}\text { CPU Time (seconds) for } \\
\text { Power Harvesting FRFs }\end{array}$ & $\begin{array}{l}\text { CPU Time (seconds) for } \\
\text { Natural Frequencies }\end{array}$ \\
\hline 5 & 0.56160 & 0.32760 \\
10 & 1.04521 & 0.57720 \\
30 & 4.27443 & 1.51321 \\
50 & 9.87486 & 2.49602 \\
\hline
\end{tabular}

Table 3. Piezoelectric material properties.

\begin{tabular}{llllll}
\hline $\begin{array}{l}\text { Reference / } \\
\text { Company }\end{array}$ & $\begin{array}{l}\text { Piezoelectric } \\
\text { material }\end{array}$ & $\begin{array}{l}\text { Young's } \\
\text { modulus } \\
\overline{\mathrm{c}}_{11}(\mathrm{GPa})\end{array}$ & $\begin{array}{l}\text { Piezoelectric } \\
\text { coefficient } \\
\mathrm{d} 31(\mathrm{pm} / \mathrm{V})\end{array}$ & $\begin{array}{l}\text { Relative } \\
\text { dielectric } \\
\text { constant } \varepsilon_{33}^{\mathrm{T}} / \varepsilon_{\mathrm{o}}\end{array}$ & $\begin{array}{l}\text { Density } \\
\left(\mathrm{kg} / \mathrm{m}^{3}\right)\end{array}$ \\
\hline Andosca, et al [16] & PVDF & 3 & 20 & 12 & 1780 \\
\hline $\begin{array}{l}\text { Piezo Systems, } \\
\text { Inc }\end{array}$ & PSI-5A4E & 66 & -190 & 1800 & 7800 \\
& PSI-5H4E & 62 & -320 & 3800 & 7800 \\
\hline $\begin{array}{l}\text { APC International, } \\
\text { Ltd }\end{array}$ & PMN-32\%PT 840 & 24.77 & -930 & 4600 & 8200 \\
\hline APC 855 & 80 & -125 & 1275 & 7600 \\
\hline $\begin{array}{l}\text { MeL Piczofine } \\
\text { Specialities }\end{array}$ & PZN-PT & 25 & -276 & 3300 & 7600 \\
\hline
\end{tabular}




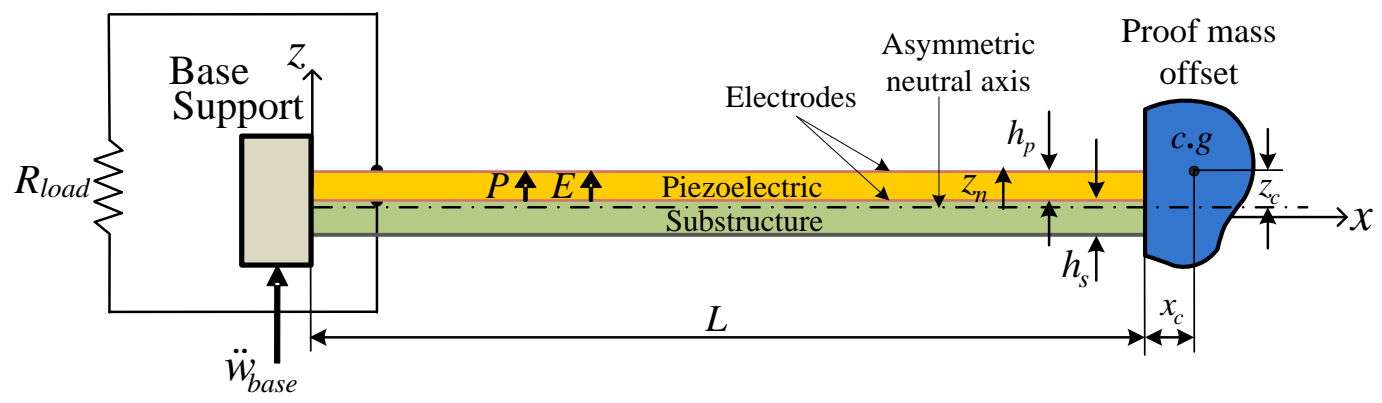

Fig. 1. Cantilevered unimorph beam structure with arbitrary proof mass offset.

(a)

(b)
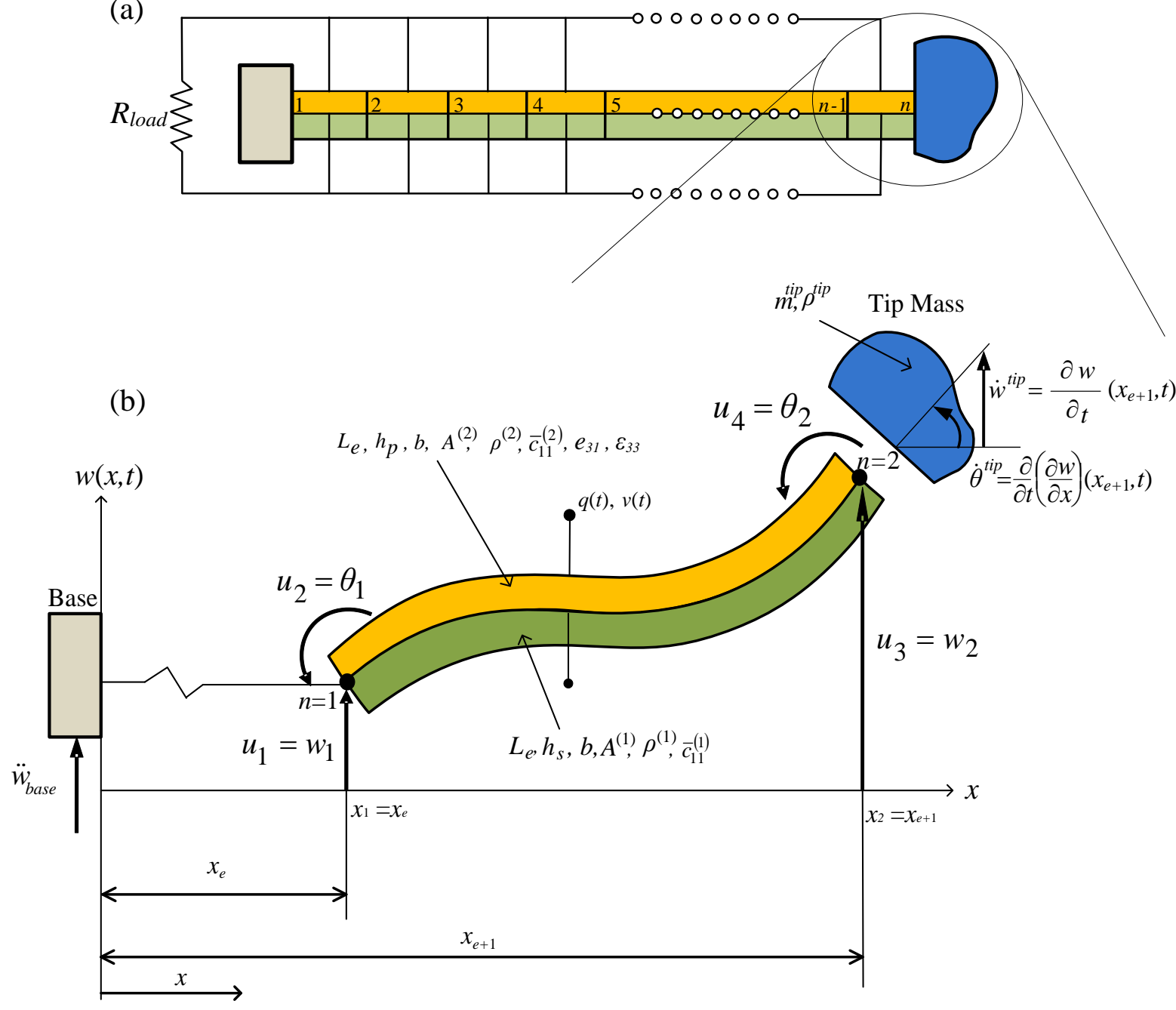

Fig. 2. (a) Electromechanical finite element discretisation and (b) Local unimorph element with an arbitrary proof mass offset at nodes $n-1$ and $n$ taking $n=2$. 


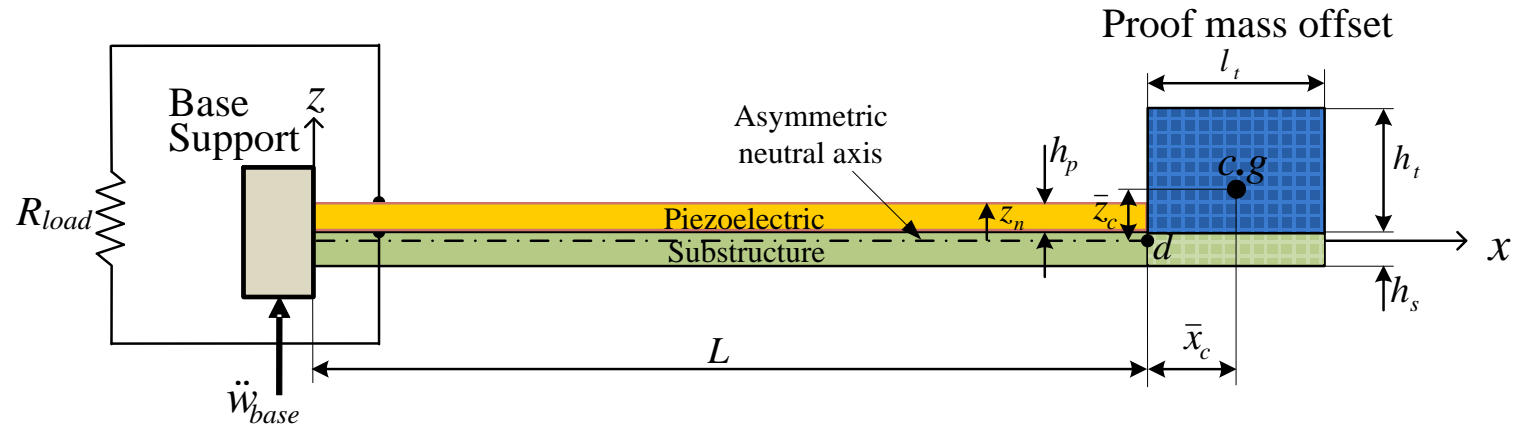

Fig. 3. Geometrical structure of unimorph beam with proof mass offset.
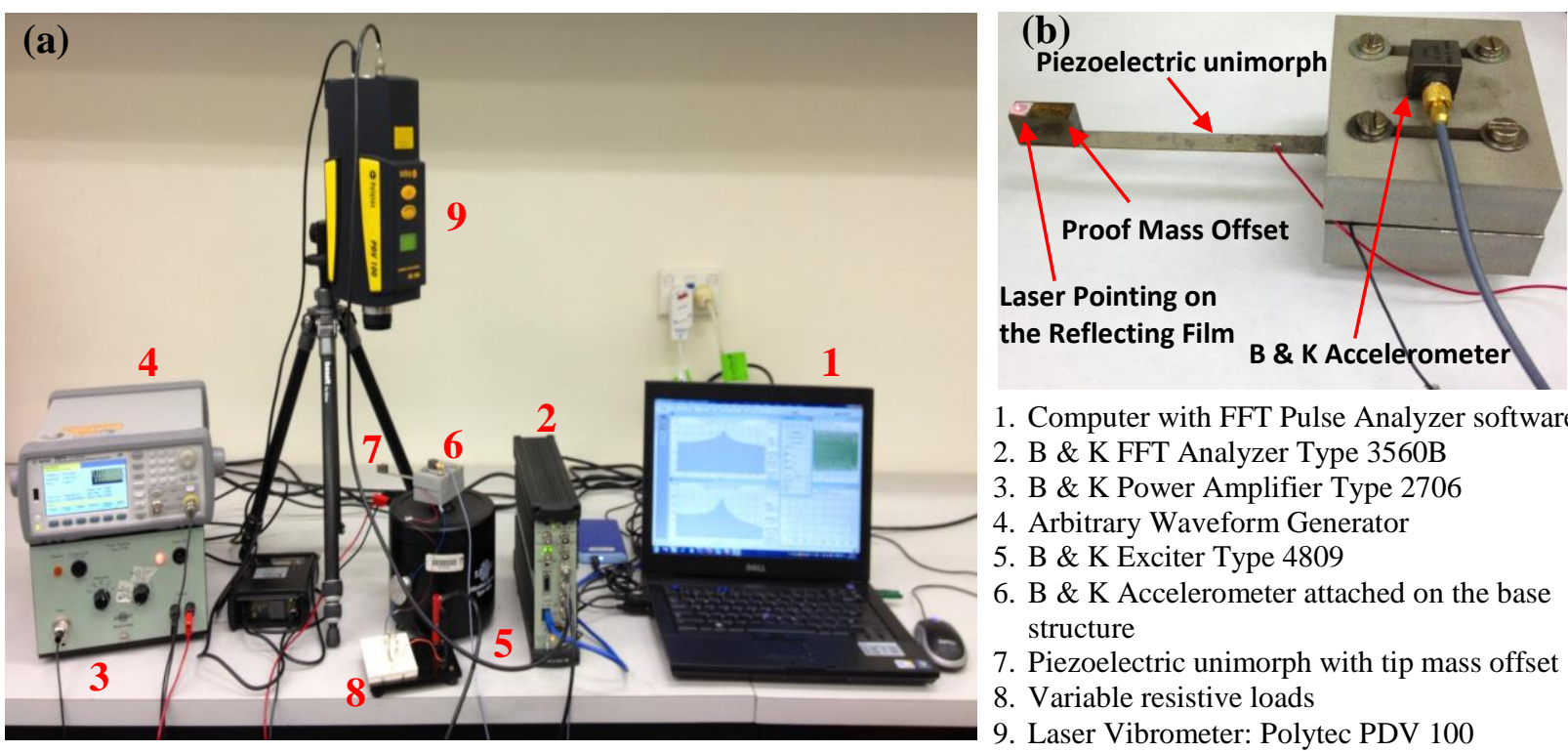

1. Computer with FFT Pulse Analyzer software

2. B \& K FFT Analyzer Type 3560B

3. B \& K Power Amplifier Type 2706

4. Arbitrary Waveform Generator

5. B \& K Exciter Type 4809

6. B \& K Accelerometer attached on the base structure

7. Piezoelectric unimorph with tip mass offset

8. Variable resistive loads

9. Laser Vibrometer: Polytec PDV 100

Fig. 4. (a) Experimental setup and (b) piezoelectric unimorph beam with proof mass offset clamped on the base structure.

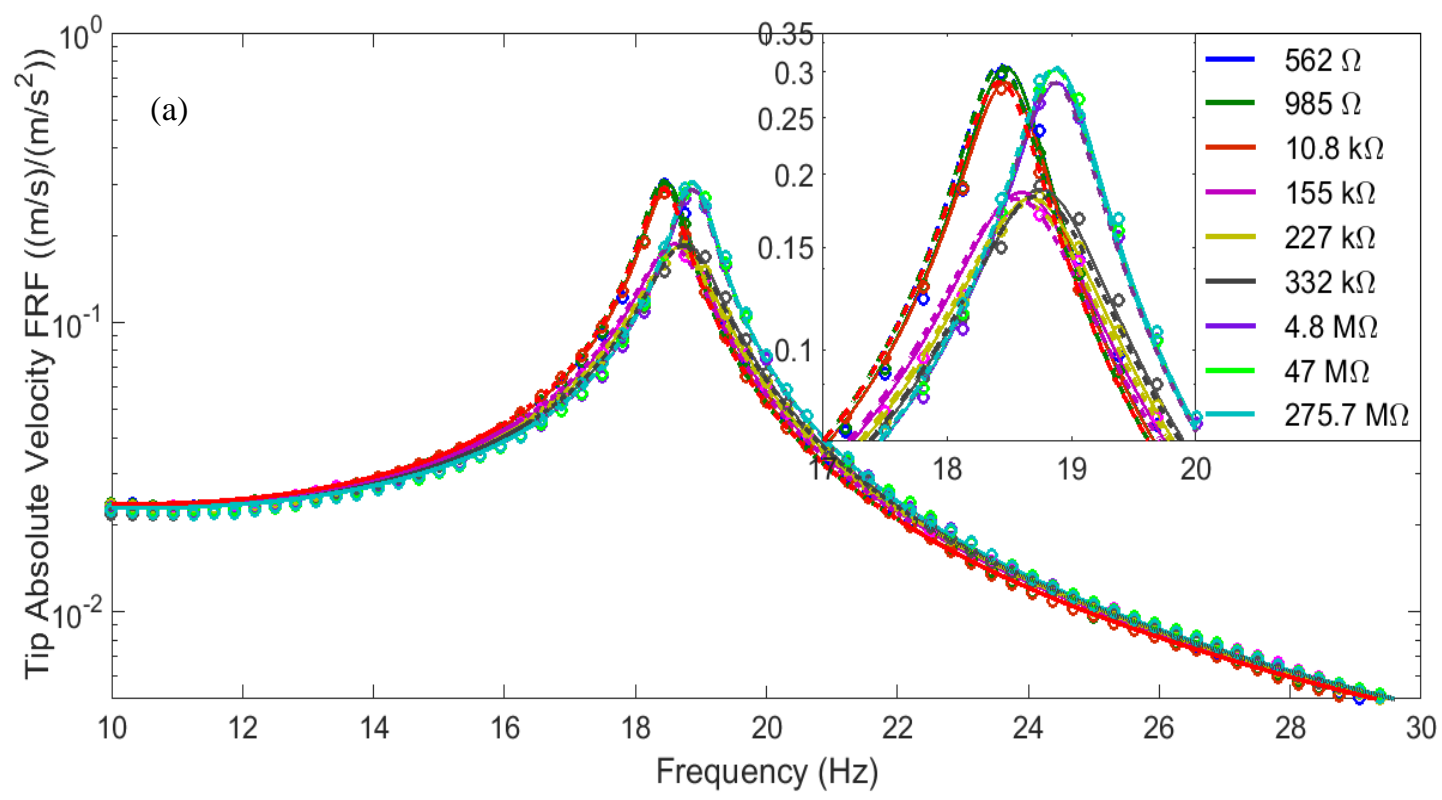



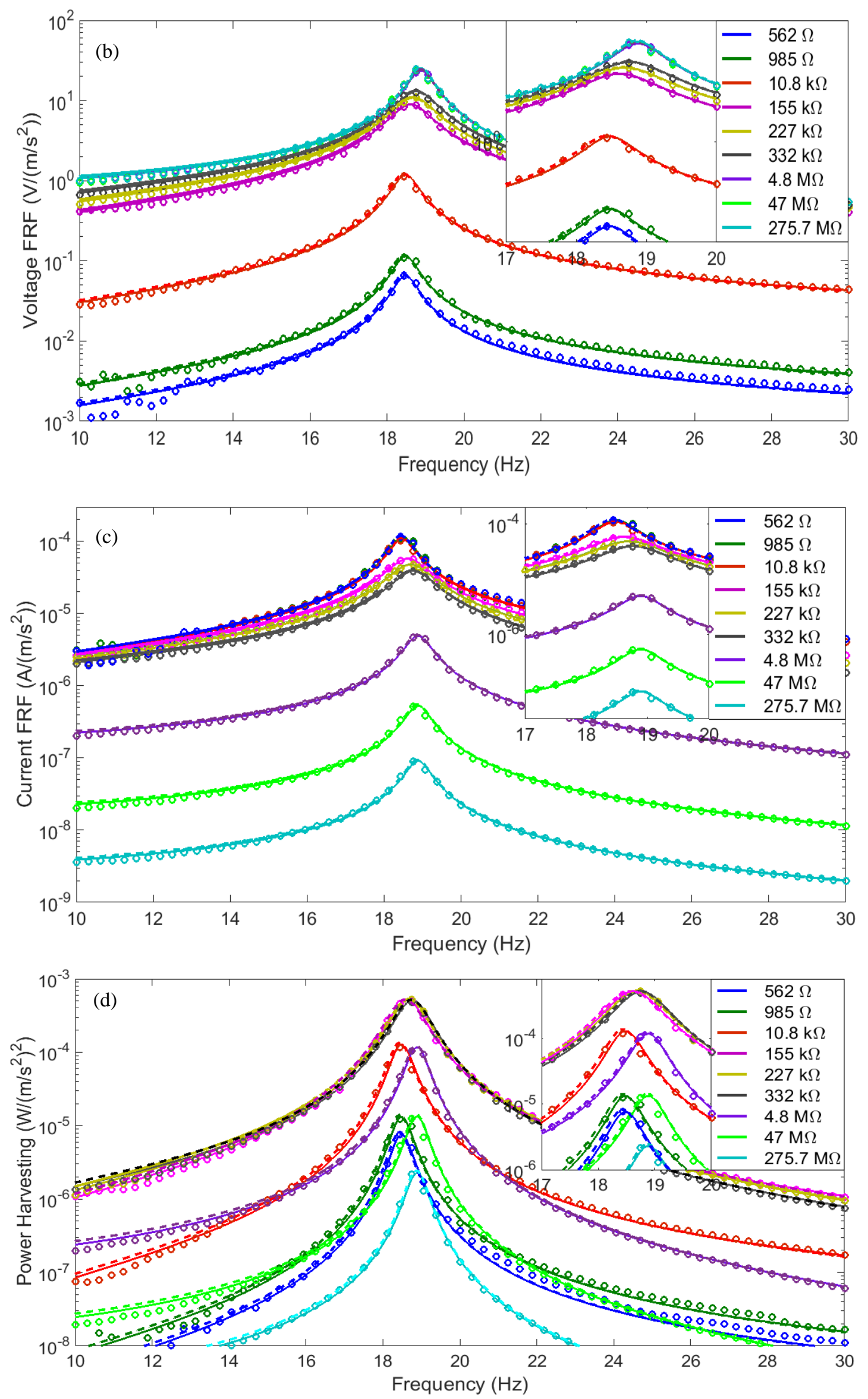

Fig. 5. Electromechanical FRFs under variable load resistances with numerical (solid lines), analytical (dash lines) and experimental results (round dot): (a) velocity, (b) voltage, (c) current and (d) power output 

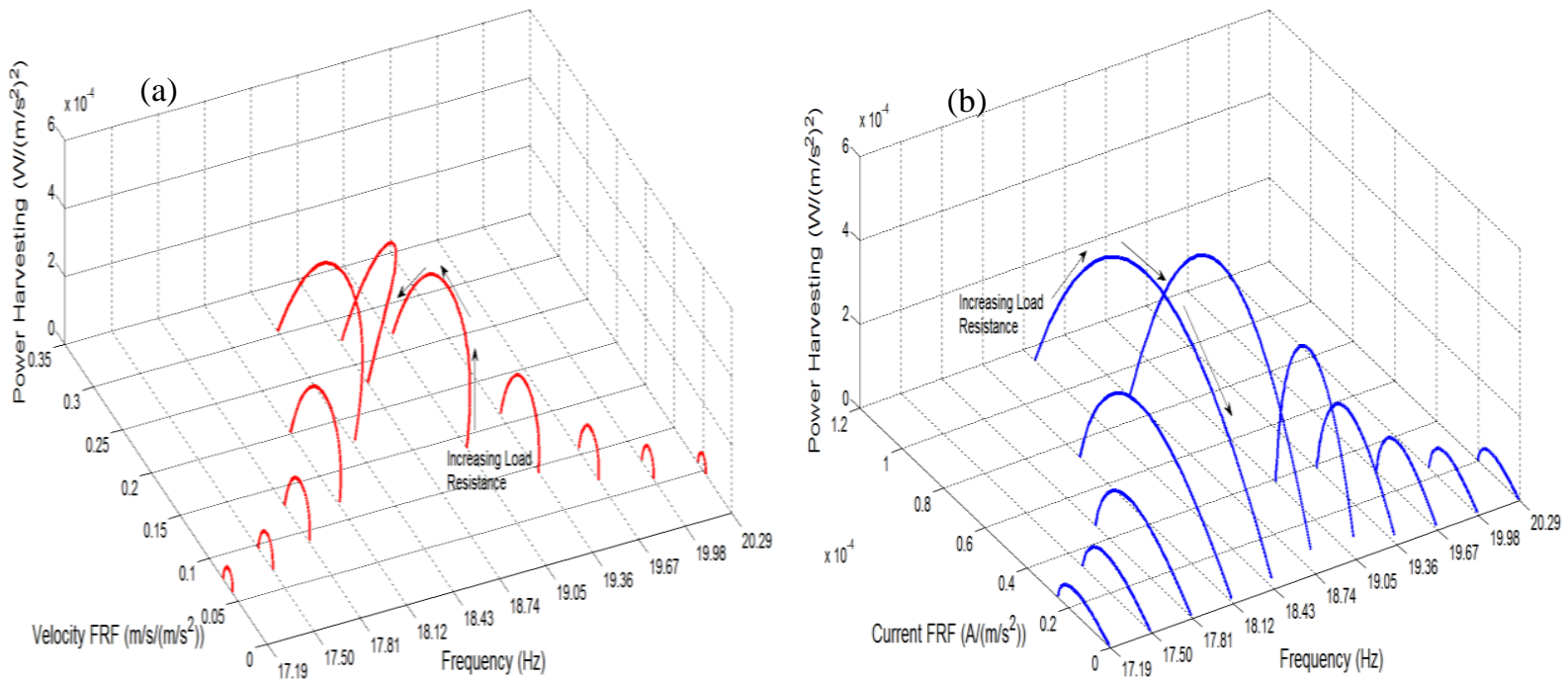

Fig. 6. (a) Frequency vs. velocity vs. optimal power output and (b) Frequency vs. current vs. optimal power output
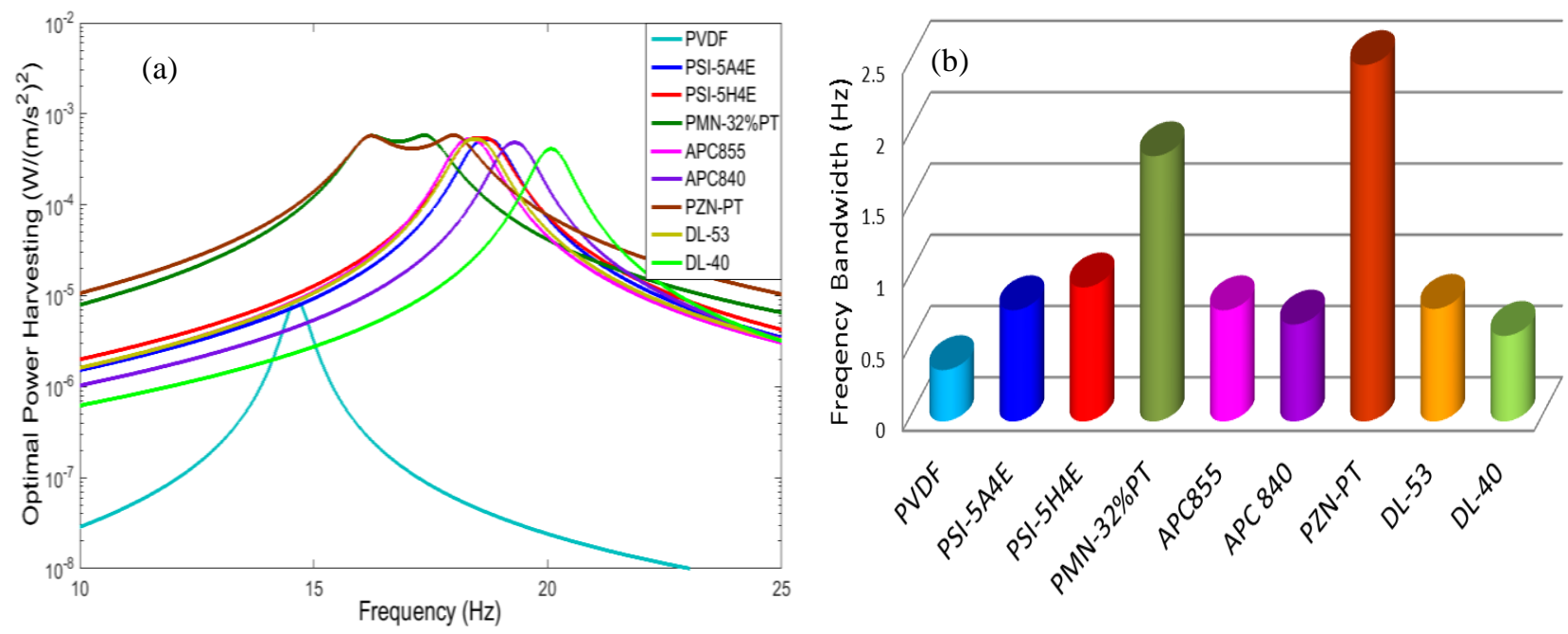

Fig. 7. Optimal responses of different piezoelectric materials with constant piezoelectric thickness of $0.127 \mathrm{~mm}$ : (a) power harvesting FRFs and (b) optimal frequency bandwidth. 

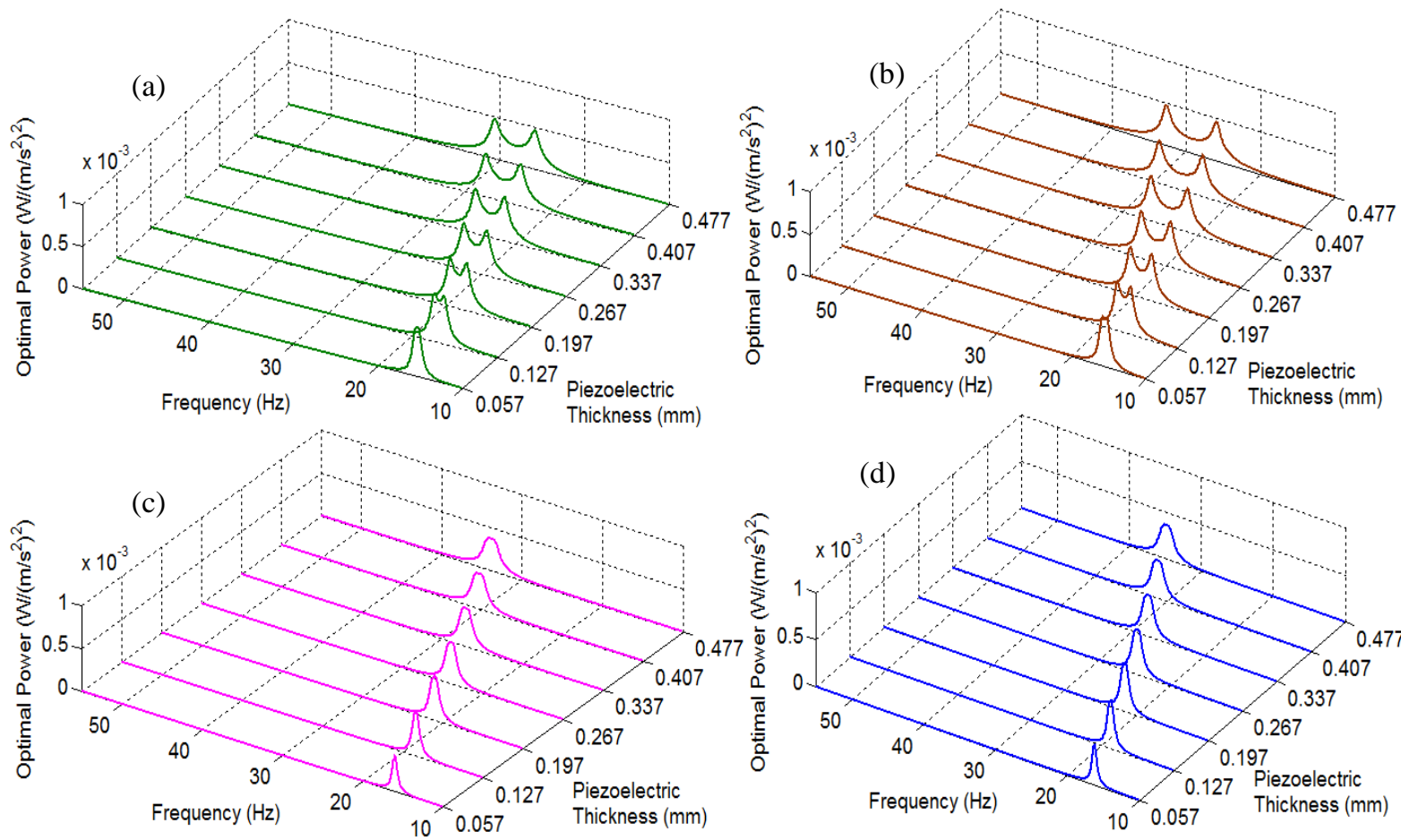

(d)

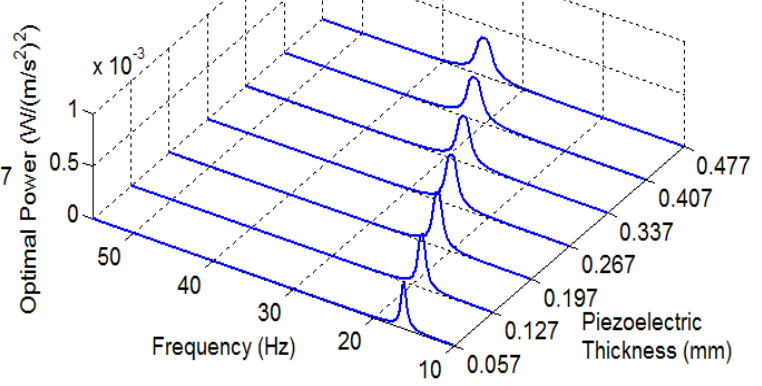

Fig. 8. Optimal responses of chosen piezoelectric materials with varying piezoelectric thickness:

(a) PMN-32\%PT, (b) PZN-PT, (c) APC855 and (d) PSI-5A4E.
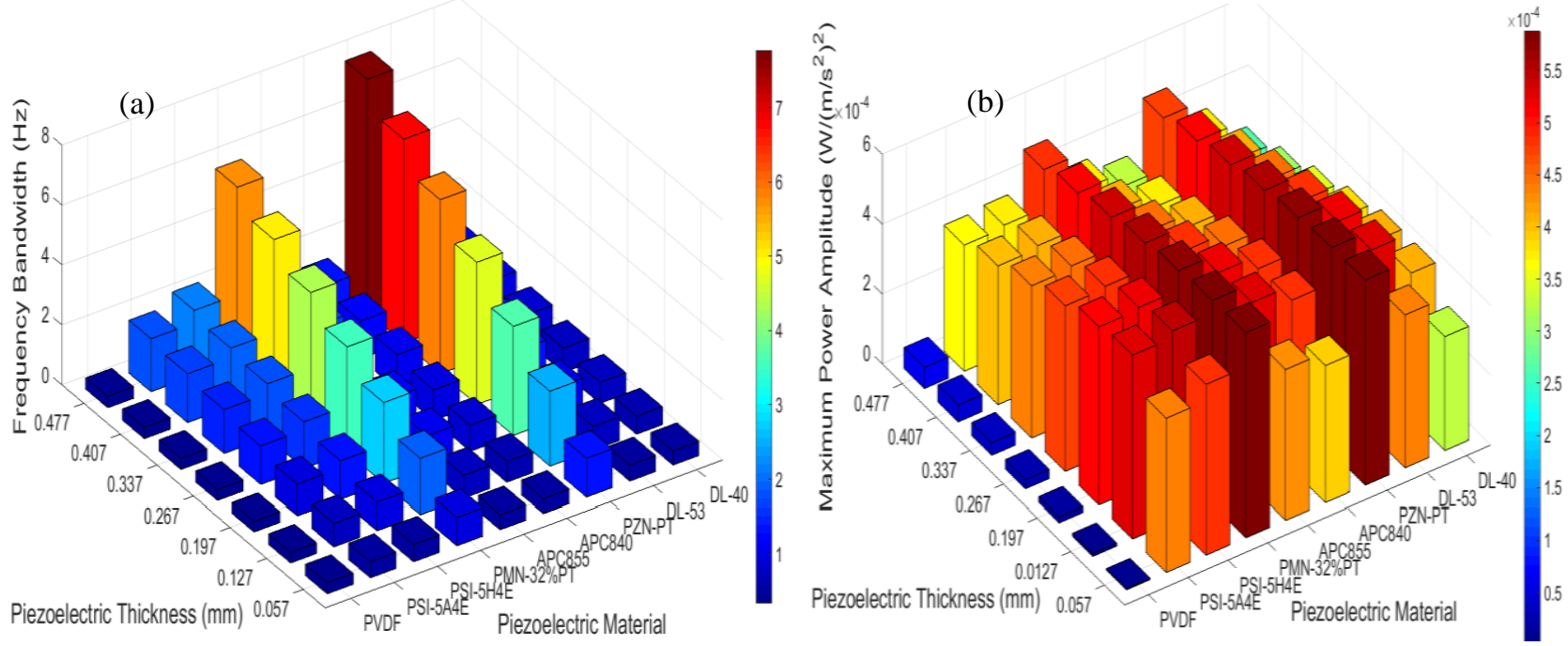

Fig. 9. Optimal responses of various piezoelectric materials with varying piezoelectric thickness:

(a) optimal frequency bandwidth and (b) power amplitude. 


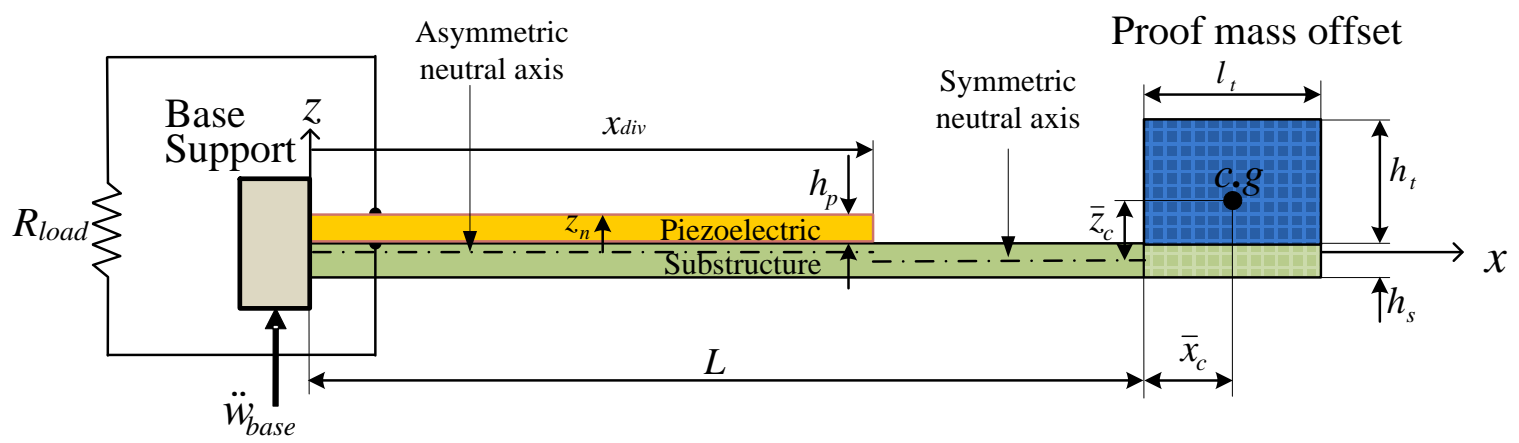

Fig. 10. Geometry of variable segmented piezoelectric coverage onto a cantilevered beam with arbitrary proof mass offset.
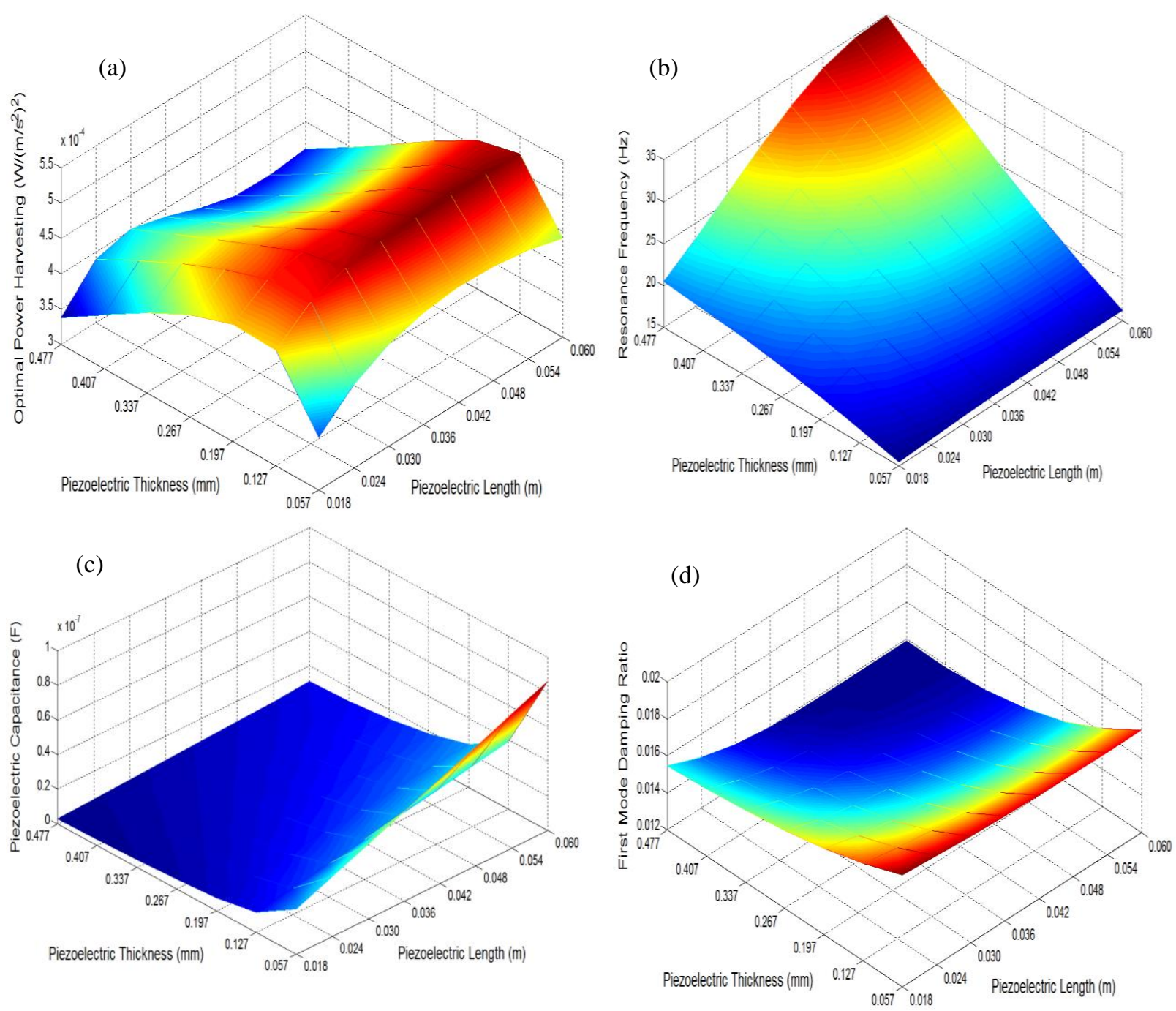

Fig. 11. Parametric optimal design of PSI-5A4E piezoelectric: (a) power harvesting FRFs, (b) resonance frequency, (c) piezoelectric capacitance and (d) damping ratio. 

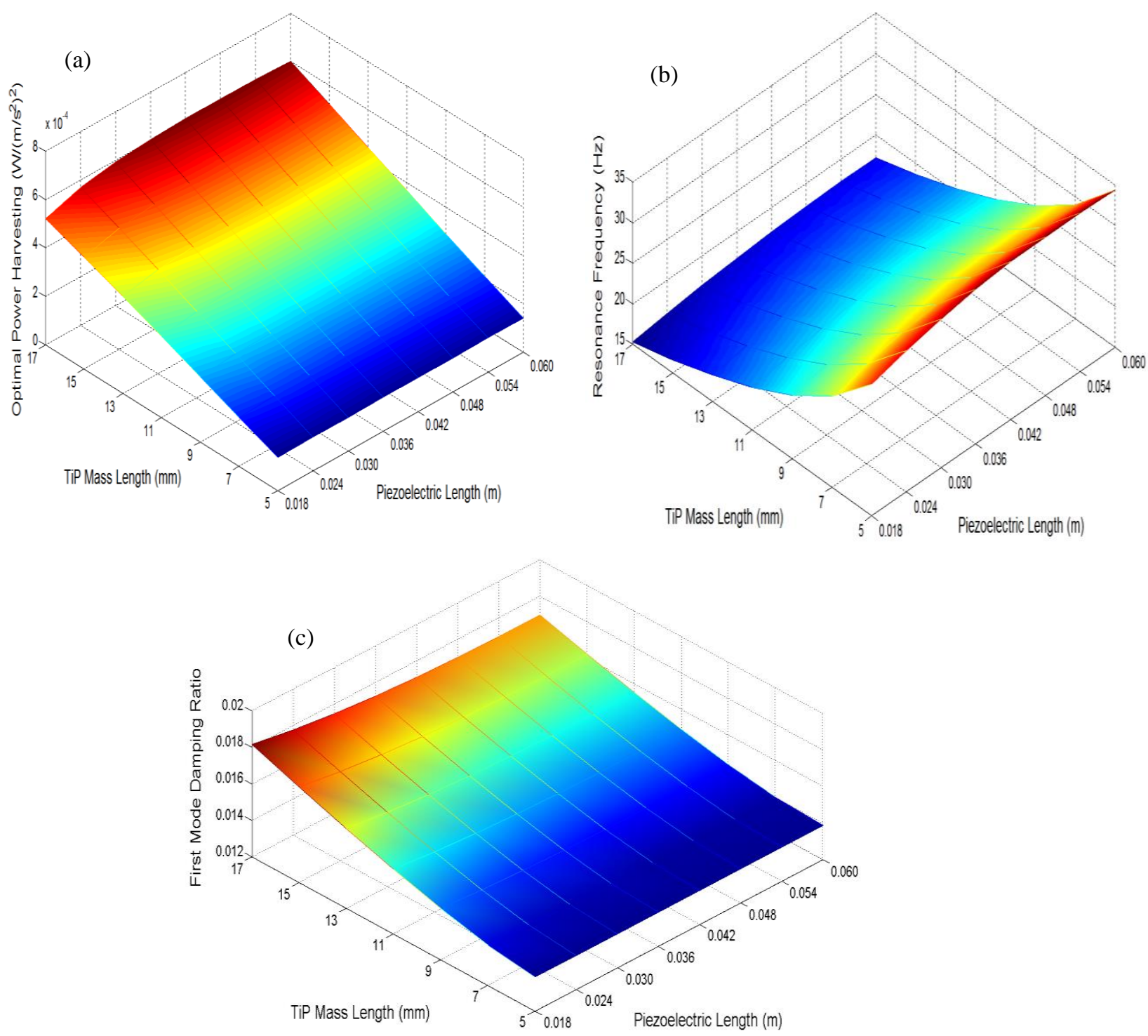

Fig. 12. Parametric optimal design of PSI-5A4E piezoelectric and proof mass offset: (a) power harvesting FRFs, (b) resonance frequency and (c) damping ratio. 